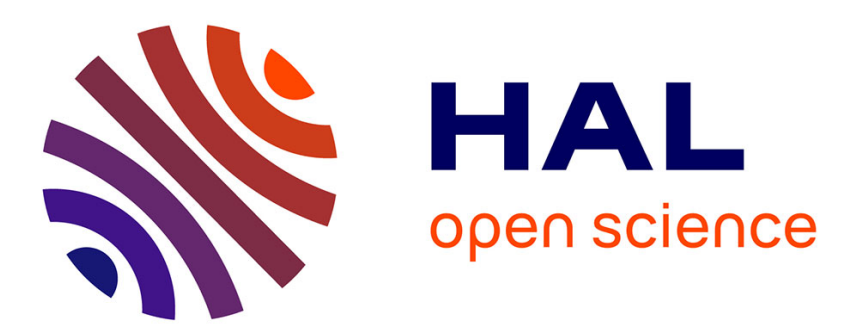

\title{
Analytical solution for waves propagation in heterogeneous acoustic/porous media. Part II: the 3D case
}

\author{
Julien Diaz, Abdelaâziz Ezziani
}

\section{- To cite this version:}

Julien Diaz, Abdelaâziz Ezziani. Analytical solution for waves propagation in heterogeneous acoustic/porous media. Part II: the 3D case. Communications in Computational Physics, 2010, 7 (3), pp.445-472. 10.4208/cicp.2009.08.149 . inria-00404228

\section{HAL Id: inria-00404228 \\ https://hal.inria.fr/inria-00404228}

Submitted on 18 Feb 2018

HAL is a multi-disciplinary open access archive for the deposit and dissemination of scientific research documents, whether they are published or not. The documents may come from teaching and research institutions in France or abroad, or from public or private research centers.
L'archive ouverte pluridisciplinaire HAL, est destinée au dépôt et à la diffusion de documents scientifiques de niveau recherche, publiés ou non, émanant des établissements d'enseignement et de recherche français ou étrangers, des laboratoires publics ou privés. 


\title{
Analytical solution for waves propagation in hetero- geneous acoustic/porous media. Part II: the 3D case
}

\author{
Julien Diaz ${ }^{1,2, *}$ and Abdelaâziz Ezziani ${ }^{2,1}$ \\ ${ }^{1}$ Project-team Magique-3D, INRIA Bordeaux Sud-Ouest \\ ${ }^{2}$ LMA Pau, CNRS UMR 5142, I.P.R.A - Université de Pau et des Pays de l'Adour, \\ avenue de l'Université BP 1155-64013 PAU CEDEX FRANCE
}

\begin{abstract}
We are interested in the modeling of wave propagation in an infinite bilayered acoustic/poroelastic media. We consider the biphasic Biot's model in the poroelastic layer. The first part was devoted to the calculation of analytical solution in two dimensions, thanks to Cagniard de Hoop method. In this second part we consider the $3 \mathrm{D}$ case.
\end{abstract}

AMS subject classifications: 34B27, 35L05, 35L15, 74F10, 74J05

Key words: Biot's model, poroelastic waves, acoustic waves, acoustic/poroelastic coupling, analytical solution, Cagniard-De Hoop's technique, 3D

\section{Introduction}

The computation of analytical solutions for wave propagation problems is of high importance for the validation of numerical computational codes or for a better understanding of the reflexion/transmission properties of the media. Cagniard-de Hoop method $[1,2]$ is a useful tool to obtain such solutions and permits to compute each type of waves ( $P$ wave, $\mathrm{S}$ wave, head wave...) independently. Although it was originally dedicated to the solution of elastodynamic wave propagation, it can be applied to any transient wave propagation problem in stratified media. However, as far as we know, few works have been dedicated to the application of this method to poroelastic medium. In [3] the analytical solution of poroelastic wave propagation in an homogeneous $2 \mathrm{D}$ medium is provided and in [4] the authors compute the analytical expression of the reflected wave at the interface between an acoustic and a poroelastic layer in two dimension but they do not explicit the expression of the transmitted waves. The coupling between acoustic and poroelastic media is of high interest for the simulation of wave propagation for seismics

*Corresponding author. Email addresses: Julien.Diaz@inria.fr (J. Diaz), Abdelaaziz.Ezziani@univ-pau.fr (A. Ezziani) 
problem in sea bottom or for ultrasound wave propagation in biological tissues, when the human skin can regarded as a fluid and the bones as a porous medium.

In order to validate computational codes of wave propagation in poroelastic media, we have implemented the codes Gar6more 2D [5] and Gar6more 3D [6] which provide the complete solution (reflected and transmitted waves) of the propagation of wave in stratified 2D or 3D media composed of acoustic/acoustic, acoustic/elastic, acoustic/poroelastic or poroelastic/poroelastic The 2D code and the 3D code are freely downloadable at

and http://www.spice-rtn.org/library/software/Gar6more2D.

$$
\text { http://www.spice-rtn.org/library/software/Gar6more3D. }
$$

In previous studies $[7,8]$ we have presented the 2D acoustic/poroelastic and poroelastic/poroelastic cases and we focus here on the 3D acoustic/poroelastic case,the 3D poroelastic/poroelastic case will be the object of forthcoming papers. We first present the model problem we want to solve and derive the Green problem from it (section 1). Then we present the analytical solution of wave propagation in a stratified 3D medium composed of an acoustic and a poroelastic layer (section 2) and we detail the computation of the solution (section 3). Finally we illustrate our results through numerical applications (section 4).

\section{The model problem}

We consider an infinite three dimensional medium $\left(\Omega=R^{3}\right)$ composed of an homogeneous acoustic layer $\left.\Omega^{+}=R^{2} \times\right] 0,+\infty\left[\right.$ and an homogeneous poroelastic layer $\Omega^{-}=$ $\left.R^{2} \times\right]-\infty, 0$ [ separated by an horizontal interface $\Gamma$ (see Fig. 1 ). We first describe the equations in the two layers ( $\$ 2.1$ and $\$ 2.2)$ and the transmission conditions on the interface $\Gamma$ (\$2.3), then we present the Green problem from which we compute the analytical solution (§2.4).

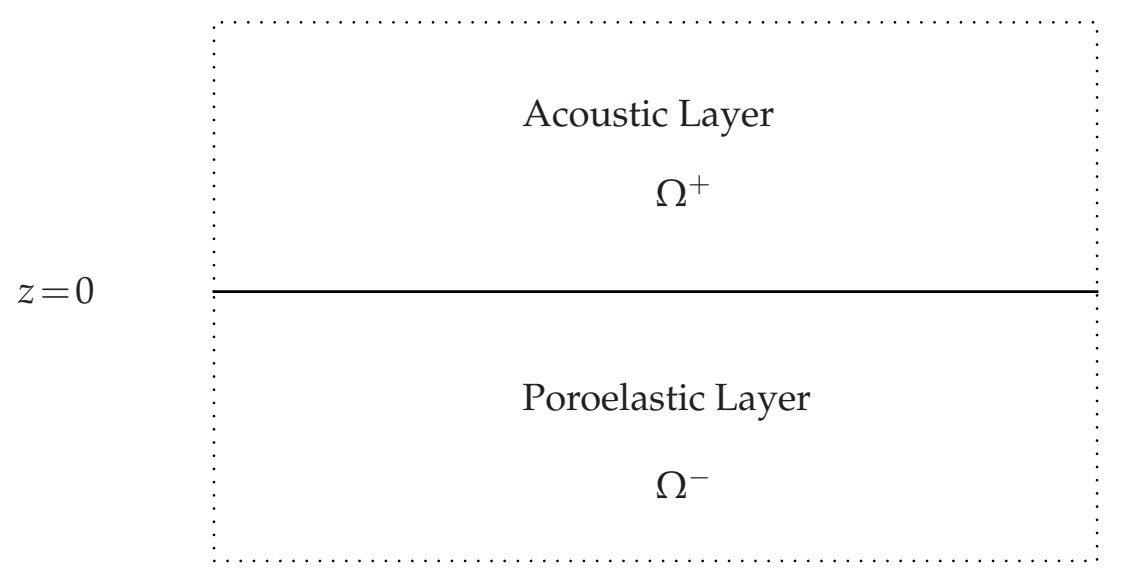

Figure 1: Configuration of the study 


\subsection{The equation of acoustics}

In the acoustic layer we consider the second order formulation of the wave equation with a point source in space, a regular source function $f$ in time and zero initial conditions:

$$
\begin{cases}\ddot{P}^{+}-V^{+2} \Delta P^{+}=\delta_{x} \delta_{y} \delta_{z-h} f(t), & \text { in } \left.\left.\Omega^{+} \times\right] 0, T\right], \\ \ddot{U}^{+}=-\frac{1}{\rho^{+}} \nabla P^{+}, & \text {in } \left.\left.\Omega^{+} \times\right] 0, T\right], \\ P^{+}(x, y, 0)=0, \dot{P}^{+}(x, y, 0)=0, & \text { in } \Omega^{+}, \\ \mathbf{U}^{+}(x, y, 0)=0, \dot{\mathbf{U}}^{+}(x, y, 0)=0, & \text { in } \Omega^{+},\end{cases}
$$

where

- $P^{+}$is the pressure;

- $\boldsymbol{U}^{+}$is the displacement field;

- $V^{+}$is the celerity of the wave;

- $\rho^{+}$is the density of the fluid.

\subsection{Biot's model}

In the second layer we consider the second order formulation of the poroelastic equations [9-11]

$$
\begin{cases}\rho^{-} \ddot{\boldsymbol{U}}_{s}^{-}+\rho_{f}^{-} \ddot{\boldsymbol{W}}^{-}-\nabla \cdot \Sigma^{-}=0, & \text { in } \left.\left.\Omega^{-} \times\right] 0, T\right], \\ \rho_{f}^{-} \ddot{\boldsymbol{U}}_{s}^{-}+\rho_{w}^{-} \ddot{\boldsymbol{W}}^{-}+\frac{1}{\mathcal{K}} \dot{\boldsymbol{W}}^{-}+\nabla P^{-}=0, & \text { in } \left.\left.\Omega^{-} \times\right] 0, T\right], \\ \Sigma^{-}=\lambda^{-} \nabla \cdot \boldsymbol{U}_{s}^{-} \boldsymbol{I}_{3}+2 \mu^{-} \varepsilon\left(\boldsymbol{U}_{s}^{-}\right)-\beta^{-} P^{-} \boldsymbol{I}_{3}, & \text { in } \left.\left.\Omega^{-} \times\right] 0, T\right], \\ \frac{1}{m^{-}} P^{-}+\beta^{-} \nabla \cdot \boldsymbol{U}_{s}^{-}+\nabla \cdot \boldsymbol{W}^{-}=0, & \text { in } \left.\left.\Omega^{-} \times\right] 0, T\right], \\ \boldsymbol{U}_{s}^{-}(x, 0)=0, \boldsymbol{W}^{-}(x, 0)=0, & \text { in } \Omega^{-}, \\ \dot{\boldsymbol{U}}_{s}^{-}(x, 0)=0, \dot{\boldsymbol{W}}^{-}(x, 0)=0, & \text { in } \Omega^{-},\end{cases}
$$

with

$$
\left(\nabla \cdot \Sigma^{-}\right)_{i}=\sum_{j=1}^{3} \frac{\partial \Sigma_{i j}^{-}}{\partial x_{j}} \forall i=1,3, I_{3} \text { is the usual identity matrix of } \mathcal{M}_{3}(\mathbb{R}),
$$

and $\varepsilon\left(\boldsymbol{U}_{s}^{-}\right)$is the solid strain tensor defined by:

$$
\varepsilon_{i j}(\boldsymbol{U})=\frac{1}{2}\left(\frac{\partial U_{i}}{\partial x_{j}}+\frac{\partial U_{j}}{\partial x_{i}}\right)
$$


In (2.2), the unknowns are:

- $\boldsymbol{U}_{s}^{-}$the displacement field of solid particles;

- $\boldsymbol{W}^{-}=\phi^{-}\left(\boldsymbol{U}_{f}^{-}-\boldsymbol{U}_{s}^{-}\right)$, the relative displacement, $\boldsymbol{U}_{f}^{-}$being the displacement field of fluid particle and $\phi^{-}$the porosity;

- $P^{-}$, the fluid pressure;

- $\Sigma^{-}$, the solid stress tensor.

The parameters describing the physical properties of the medium are given by:

- $\rho^{-}=\phi^{-} \rho_{f}^{-}+\left(1-\phi^{-}\right) \rho_{s}^{-}$is the overall density of the saturated medium, with $\rho_{s}^{-}$the density of the solid and $\rho_{f}^{-}$the density of the fluid;

- $\rho_{w}^{-}=a^{-} \rho_{f}^{-} / \phi^{-}$, where $a^{-}$the tortuosity of the solid matrix;

- $\mathcal{K}^{-}=\mathcal{K}^{-} / \eta^{-}, \mathcal{K}^{-}$is the permeability of the solid matrix and $\eta$ is the viscosity of the fluid:

- $m^{-}$and $\beta^{-}$are positive physical coefficients: $\beta^{-}=1-K_{b}^{-} / K_{s}^{-}$ and $m^{-}=\left[\phi^{-} / K_{f}^{-}+\left(\beta^{-}-\phi^{-}\right) / K_{s}^{-}\right]^{-1}$, where $K_{s}^{-}$is the bulk modulus of the solid, $K_{f}^{-}$is the bulk modulus of the fluid and $K_{b}^{-}$is the frame bulk modulus;

- $\mu^{-}$is the frame shear modulus, and $\lambda^{-}=K_{b}^{-}-2 \mu^{-} / 3$ is the Lamé constant.

\subsection{Transmission conditions}

Let $n$ be the unitary normal vector of $\Gamma$ outwardly directed to. The transmission conditions on the interface between the acoustic and porous medium are [12] :

$$
\left\{\begin{array}{l}
\boldsymbol{W}^{-} \cdot \boldsymbol{n}=\left(\boldsymbol{U}^{+}-\boldsymbol{U}_{s}^{-}\right) \cdot \boldsymbol{n}, \\
P^{-}=P^{+}, \\
\Sigma^{-} \boldsymbol{n}=-P^{+} \boldsymbol{n} .
\end{array}\right.
$$

\subsection{The Green problem}

We won't compute directly the solution to $(2.1-2.2-2.3)$ but the solution to the following Green problem:

$$
\begin{array}{ll}
\ddot{p}^{+}-V^{+2} \Delta p^{+}=\delta_{x} \delta_{y} \delta_{z-h} \delta_{t}, & \text { in } \left.\left.\Omega^{+} \times\right] 0, T\right], \\
\ddot{u}^{+}=-\frac{1}{\rho^{+}} \nabla p^{+}, & \text {in } \left.\left.\Omega^{+} \times\right] 0, T\right],
\end{array}
$$




$$
\begin{array}{cl}
\rho^{-} \ddot{\boldsymbol{u}}_{s}^{-}+\rho_{f}^{-} \ddot{\boldsymbol{w}}^{-}-\nabla \cdot \sigma^{-}=0, & \text { in } \left.\left.\Omega^{-} \times\right] 0, T\right], \\
\rho_{f}^{-} \ddot{\boldsymbol{u}}_{s}^{-}+\rho_{w}^{-} \ddot{\boldsymbol{w}}^{-}+\frac{1}{\mathcal{K}} \dot{\boldsymbol{w}}^{-}+\nabla p^{-}=0, & \text { in } \left.\left.\Omega^{-} \times\right] 0, T\right], \\
\sigma^{-}=\lambda^{-} \nabla \cdot \boldsymbol{u}_{s}^{-} \boldsymbol{I}_{3}+2 \mu^{-} \varepsilon\left(\boldsymbol{u}_{s}^{-}\right)-\beta^{-} p^{-} \boldsymbol{I}_{3}, & \text { in } \left.\left.\Omega^{-} \times\right] 0, T\right], \\
\frac{1}{m^{-}} p^{-}+\beta^{-} \nabla \cdot \boldsymbol{u}_{s}^{-}+\nabla \cdot \boldsymbol{w}^{-}=0, & \text { in } \left.\left.\Omega^{-} \times\right] 0, T\right], \\
\boldsymbol{w}^{-} \cdot \boldsymbol{n}=\left(\boldsymbol{u}^{+}-\boldsymbol{u}_{s}^{-}\right) \cdot \boldsymbol{n}, & \\
p^{-}=p^{+}, & \text {on } \Gamma, \\
\sigma^{-} \boldsymbol{n}=-p^{+} \boldsymbol{n}, & \text { on } \Gamma,
\end{array}
$$

The solution to $(2.1-2.2-2.3)$ is then computed from the solution of the Green Problem thanks to a convolution by the source function. For instance we have :

$$
P^{+}(x, y, t)=p^{+}(x, y, .) * f(.)=\int_{0}^{t} p^{+}(x, y, \tau) f(t-\tau) d \tau
$$

(we have similar relations for the other unknowns). We also suppose that the poroelastic medium is non dissipative, i.e the viscosity $\eta^{-}=0$. Using the equations (2.5c, $\left.2.5 \mathrm{~d}\right)$ we can eliminate $\sigma^{-}$and $p^{-}$in (2.5) and we obtain the the equivalent system:

$$
\begin{cases}\rho^{-} \ddot{\boldsymbol{u}}_{s}^{-}+\rho_{f}^{-} \ddot{\boldsymbol{w}}^{-}-\alpha^{-} \nabla\left(\nabla \cdot \boldsymbol{u}_{s}^{-}\right)+\mu^{-} \nabla \times\left(\nabla \times \boldsymbol{u}_{s}^{-}\right)-m^{-} \beta^{-} \nabla\left(\nabla \cdot \boldsymbol{w}^{-}\right)=0, & z<0, \\ \rho_{f}^{-} \ddot{\boldsymbol{u}}_{s}^{-}+\rho_{w}^{-} \ddot{\boldsymbol{w}}^{-}-m^{-} \beta^{-} \nabla\left(\nabla \cdot \boldsymbol{u}_{s}^{-}\right)-m^{-} \nabla\left(\nabla \cdot \boldsymbol{w}^{-}\right)=0, & z<0,\end{cases}
$$

with $\alpha^{-}=\lambda^{-}+2 \mu^{-}+m^{-} \beta^{-2}$.

And using the equation (2.4b) the transmission conditions (2.6) on $z=0$ are rewritten as:

$$
\begin{aligned}
& \ddot{u}_{s z}^{-}+\ddot{w}_{z}^{-}=-\frac{1}{\rho^{+}} \partial_{z} p^{+}, \\
& -m^{-} \beta^{-} \nabla \cdot \boldsymbol{u}_{s}^{-}-m^{-} \nabla \cdot \boldsymbol{w}^{-}=p^{+}, \\
& \partial_{z} u_{s x}^{-}+\partial_{x} u_{s z}^{-}=0, \\
& \partial_{z} u_{s y}^{-}+\partial_{y} u_{s z}^{-}=0, \\
& \left(\lambda^{-}+m^{-} \beta^{-2}\right) \nabla \cdot \boldsymbol{u}_{s}^{-}+2 \mu^{-} \partial_{z} u_{s z}^{-}+m^{-} \beta^{-} \nabla \cdot \boldsymbol{w}=-p^{+} .
\end{aligned}
$$


We split the displacement fields $\boldsymbol{u}_{s}^{-}$and $\boldsymbol{w}^{-}$into irrotationnal and isovolumic fields (Pwave and S-wave):

$$
\boldsymbol{u}_{s}^{-}=\nabla \Theta_{u}^{-}+\nabla \times \mathbf{\Psi}_{u}^{-} ; w^{-}=\nabla \Theta_{w}^{-}+\nabla \times \mathbf{\Psi}_{w}^{-} .
$$

The vectors $\Psi_{u}^{-}$and $\Psi_{w}^{-}$are not uniquely defined since:

$$
\nabla \times\left(\mathbf{\Psi}_{\ell}^{-}+\nabla C\right)=\nabla \times \mathbf{\Psi}_{\ell}^{-}, \forall \ell \in\{u, w\},
$$

for all scalar field $C$. To define a unique $\Psi_{\ell}^{-}$we impose the gauge condition:

$$
\nabla \cdot \Psi_{\ell}^{-}=0 .
$$

The vectorial space of $\Psi_{\ell}^{-}$verifying this last condition is written as:

$$
\Psi_{\ell}^{-}=\left[\begin{array}{c}
\partial_{y} \\
-\partial_{x} \\
0
\end{array}\right] \Psi_{\ell, 1}^{-}+\left[\begin{array}{c}
\partial_{x z}^{2} \\
\partial_{y z}^{2} \\
-\partial_{x x}^{2}-\partial_{y y}^{2}
\end{array}\right] \Psi_{\ell, 2^{\prime}}^{-}
$$

where $\Psi_{\ell, 1}^{-}$and $\Psi_{\ell, 2}^{-}$are two scalar fields. The displacement fields $\boldsymbol{u}_{s}^{-}$and $\boldsymbol{w}^{-}$are written in the form:

$$
\begin{gathered}
\boldsymbol{u}_{s}^{-}=\nabla \Theta_{u}^{-}+\left[\begin{array}{c}
\partial_{x z}^{2} \\
\partial_{y z}^{2} \\
-\partial_{x x}^{2}-\partial_{y y}^{2}
\end{array}\right] \Psi_{u, 1}^{-}-\left[\begin{array}{c}
\partial_{y} \\
-\partial_{x} \\
0
\end{array}\right] \Delta \Psi_{u, 2}^{-}, \\
\boldsymbol{w}^{-}=\nabla \Theta_{w}^{-}+\left[\begin{array}{c}
\partial_{x z}^{2} \\
\partial_{y z}^{2} \\
-\partial_{x x}^{2}-\partial_{y y}^{2}
\end{array}\right] \Psi_{w, 1}^{-}-\left[\begin{array}{c}
\partial_{y} \\
-\partial_{x} \\
0
\end{array}\right] \Delta \Psi_{w, 2}^{-} .
\end{gathered}
$$

Using this last change of variables, we can then rewrite the system (2.7) in the following form:

$$
\begin{cases}A \ddot{\Theta}^{-}-B \Delta \Theta^{-}=0, & z<0, \\ \ddot{\Psi}_{u, 1}^{-}-V_{S}^{-2} \Delta \Psi_{u, 1}^{-}=0, & z<0, \\ \ddot{\Psi}_{u, 2}^{-}-V_{S}^{-2} \Delta \Psi_{u, 2}^{-}=0, & z<0, \\ \ddot{\Psi}_{w}^{-}=-\frac{\rho_{f}^{-}}{\rho_{w}^{-}} \ddot{\Psi}_{u}^{-}, & z<0,\end{cases}
$$

where $\Theta^{-}=\left(\Theta_{u}^{-}, \Theta_{w}^{-}\right)^{t}, A$ and $B$ are $2 \times 2$ symmetric matrices:

$$
A=\left(\begin{array}{cc}
\rho^{-} & \rho_{f}^{-} \\
\rho_{f}^{-} & \rho_{w}^{-}
\end{array}\right) ; B=\left(\begin{array}{cc}
\lambda^{-}+2 \mu^{-}+m^{-}\left(\beta^{-}\right)^{2} & m^{-} \beta^{-} \\
m^{-} \beta^{-} & m^{-}
\end{array}\right)
$$


and

$$
V_{S}^{-}=\sqrt{\frac{\mu \rho_{w}^{-}}{\rho^{-} \rho_{w}^{-}-\rho_{f}^{-2}}}
$$

is the S-wave velocity.

We multiply the first equation of the system (2.11) by the inverse of $A$. The matrix $A^{-1} B$ is diagonalizable: $A^{-1} B=\mathcal{P} D \mathcal{P}^{-1}$, where $\mathcal{P}$ is the change-of-coordinate matrix, $D=\operatorname{diag}\left(V_{P f}^{-2}, V_{P s}^{-2}\right)$ is the diagonal matrix similar to $A^{-1} B, V_{P f}^{-}$and $V_{P s}^{-}$are respectively the fast $\mathrm{P}$-wave velocity and the slow $\mathrm{P}$-wave velocity $\left(V_{P_{s}}<V_{P f}\right)$.

Using the change of variables

$$
\Phi^{-}=\left(\Phi_{P f}^{-}, \Phi_{P s}^{-}\right)^{t}=\mathcal{P}^{-1} \Theta^{-},
$$

we obtain the uncoupled system on fast P-waves, slow P-waves and S-waves:

$$
\begin{cases}\ddot{\Phi}^{-}-D \Delta \Phi^{-}=0, & z<0 \\ \ddot{\Psi}_{u, i}^{-}-V_{S}^{-2} \Delta \Psi_{u, i}^{-}=0, i=1,2, & z<0, \\ \Psi_{w}^{-}=-\frac{\rho_{f}^{-}}{\rho_{w}^{-}} \Psi_{u}^{-} . & z<0 .\end{cases}
$$

Using the transmission conditions $(2.8 \mathrm{c})-(2.8 \mathrm{~d})$, we obtain:

$$
\begin{array}{lr}
2 \partial_{x z}^{2} \Theta_{u}^{-}+\partial_{x}\left(\partial_{z z}^{2}-\Delta_{\perp}\right) \Psi_{u, 1}^{-}-\partial_{y z}^{2} \Delta \Psi_{u, 2}^{-}=0, & \text { on } \Gamma, \\
2 \partial_{y z}^{2} \Theta_{u}^{-}+\partial_{y}\left(\partial_{z z}^{2}-\Delta_{\perp}\right) \Psi_{u, 1}^{-}+\partial_{x z}^{2} \Delta \Psi_{u, 2}^{-}=0, & \text { on } \Gamma,
\end{array}
$$

with $\Delta_{\perp}=\partial_{x x}^{2}+\partial_{y y}^{2}$. Applying the derivative $\partial_{y}$ to the equation (2.14a), $\partial_{x}$ to the equation $(2.14 \mathrm{~b})$ and subtracting the first obtained equation from the second one, we get:

$$
\left(\partial_{z} \Delta_{\perp}\right) \Delta \Psi_{u, 2}^{-}=0, \quad \text { on } \Gamma,
$$

moreover, using that $\Psi_{u, 2}^{-}$satisfies the wave equation:

$$
\ddot{\Psi}_{u, 2}^{-}-V_{S}^{-2} \Delta \Psi_{u, 2}^{-}=0, z<0,
$$

and that $\boldsymbol{u}_{s}^{-}$and $\boldsymbol{w}^{-}$satisfy, at $t=0, \boldsymbol{u}_{s}^{-}=\dot{\boldsymbol{u}}_{s}^{-}=\boldsymbol{w}^{-}=\dot{\boldsymbol{w}}^{-}=0$, we obtain:

$$
\Psi_{u, 2}^{-}=0, z \leq 0,
$$

and from (2.14) we deduce the transmission condition equivalent to (2.8c) and $(2.8 \mathrm{~d})$ :

$$
2 \partial_{z}^{2} \Theta_{u}^{-}+\left(\partial_{z z}^{2}-\Delta_{\perp}\right) \Psi_{u, 1}^{-}=0, \quad \text { on } \Gamma .
$$


Finally, we obtain the Green problem equivalent to $(2.4,2.5,2.6)$ :

$$
\begin{cases}\ddot{p}^{+}-V^{+2} \Delta p^{+}=\delta_{x} \delta_{y} \delta_{z-h} \delta_{t}, & z>0 \\ \ddot{\Phi}_{i}^{-}-V_{i}^{-2} \Delta \Phi_{i}^{-}=0, \quad i \in\left\{P f, P_{S}, S\right\}, & z<0 \\ \mathcal{B}\left(p^{+}, \Phi_{P f}^{-}, \Phi_{P_{S}}^{-} \Phi_{S}^{-}\right)=0, & z=0\end{cases}
$$

where we have set $\Phi_{S}^{-}=\Psi_{u, 1}^{-}$in order to have similar notations for the $P f, P s$ and $S$ waves. The operator $\mathcal{B}$ represents the transmission conditions on $\Gamma$ :

$$
\mathcal{B}\left(\begin{array}{c}
p^{+} \\
\Phi_{P f}^{-} \\
\Phi_{P S}^{-} \\
\Phi_{S}^{-}
\end{array}\right)=\left[\begin{array}{cccc}
\frac{1}{\rho^{+}} \partial_{z} & \left(\mathcal{P}_{11}+\mathcal{P}_{21}\right) \partial_{z t t}^{3} & \left(\mathcal{P}_{12}+\mathcal{P}_{22}\right) \partial_{z t t}^{3} & \left(\frac{\rho_{f}^{-}}{\rho_{w}^{-}}-1\right) \partial_{t t}^{2} \Delta_{\perp} \\
1 & \frac{m^{-}\left(\beta^{-} \mathcal{P}_{11}+\mathcal{P}_{21}\right)}{V_{P f}^{-2}} \partial_{t t}^{2} & \frac{m^{-}\left(\beta^{-} \mathcal{P}_{12}+\mathcal{P}_{22}\right)}{V_{P s}^{-2}} \partial_{t t}^{2} & 0 \\
0 & 2 \mathcal{P}_{11} \partial_{z} & 2 \mathcal{P}_{12} \partial_{z} & \partial_{z z}^{2}-\Delta_{\perp} \\
1 & \mathcal{B}_{42} & \mathcal{B}_{43} & -2 \mu^{-} \partial_{z} \Delta_{\perp}
\end{array}\right]\left[\begin{array}{c}
p^{+} \\
\Phi_{P f}^{-} \\
\Phi_{P S}^{-} \\
\Phi_{S}^{-}
\end{array}\right]
$$

where $\mathcal{P}_{i j}, i, j=1,2$ are the components of the change-of-coordinates matrix $\mathcal{P}, \mathcal{B}_{42}$ and $\mathcal{B}_{43}$ are given by:

$$
\begin{aligned}
& \mathcal{B}_{42}=\frac{\left(\lambda^{-}+m^{-} \beta^{-2}\right) \mathcal{P}_{11}+m^{-} \beta^{-} \mathcal{P}_{21}}{V_{P f}^{-2}} \partial_{t t}^{2}+2 \mu^{-} \mathcal{P}_{11} \partial_{z z}^{2} \\
& \mathcal{B}_{43}=\frac{\left(\lambda^{-}+m^{-} \beta^{-2}\right) \mathcal{P}_{12}+m^{-} \beta^{-} \mathcal{P}_{22}}{V_{P s}^{-2}} \partial_{t t}^{2}+2 \mu^{-} \mathcal{P}_{12} \partial_{z z}^{2} .
\end{aligned}
$$

To obtain this operator we have used the transmission conditions $(2.8 \mathrm{a}, 2.8 \mathrm{~b}, 2.16,2.8 \mathrm{e})$, the change of variables (2.9) and the uncoupled system (2.13).

Moreover, we can determine the solid displacement $\boldsymbol{u}_{s}^{-}$by using the change of variables (2.9) and the fluid displacement $\boldsymbol{u}^{+}$by using (2.4b).

\section{Expression of the analytical solution}

Since the problem is invariant by a rotation around the $z$-axis, we will only consider the case $y=0$ and $x>0$, so that the y-component of all the displacements are zero. The 
solution for $y \neq 0$ or $x \leq 0$ is deduced from the solution for $y=0$ by the relations

$$
\begin{aligned}
& p(x, y, z, t)=p\left(\sqrt{x^{2}+y^{2}}, 0, z, t\right), \\
& u_{s x}(x, y, z, t)=\frac{x}{\sqrt{x^{2}+y^{2}}} u_{s x}\left(\sqrt{x^{2}+y^{2}}, 0, z, t\right), \\
& u_{s y}(x, y, z, t)=\frac{y}{\sqrt{x^{2}+y^{2}}} u_{s x}\left(\sqrt{x^{2}+y^{2}}, 0, z, t\right), \\
& u_{s z}(x, y, z, t)=u_{s z}\left(\sqrt{x^{2}+y^{2}}, 0, z, t\right) .
\end{aligned}
$$

To state our results, we need the following notations and definitions:

1. Definition of the complex square root. For $q_{x} \in \mathbb{C} \backslash \mathbb{R}^{-}$, we use the following definition of the square root $g\left(q_{x}\right)=q_{x}^{1 / 2}$ :

$$
g\left(q_{x}\right)^{2}=q_{x} \quad \text { and } \quad \Re e\left[g\left(q_{x}\right)\right]>0 .
$$

The branch cut of $g\left(q_{x}\right)$ in the complex plane will thus be the half-line defined by $\left\{q_{x} \in \mathbb{R}^{-}\right\}$(see Fig. 2). In the following, we'll use the abuse of notation $g\left(q_{x}\right)=$ $\mathrm{i} \sqrt{-q_{x}}$ for $q_{x} \in \mathbb{R}^{-}$.

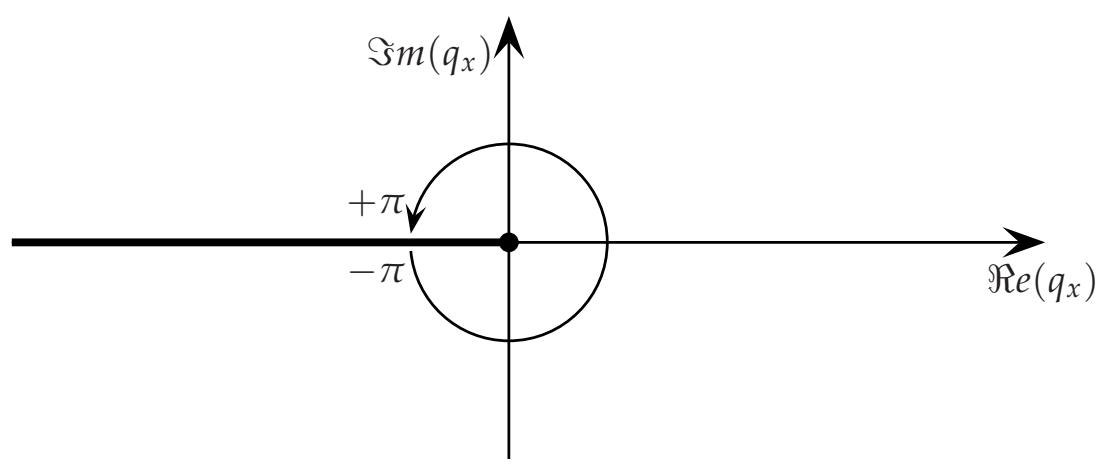

Figure 2: Definition of the function $x \mapsto(x)^{1 / 2}$

2. Definition of the fictitious velocities For a given $q \in \mathbb{R}$, we define the fictitious velocities $\mathcal{V}^{+}(q)$ and $\mathcal{V}_{i}^{-}(q)$ for $i \in\left\{P f, P_{S}, S\right\}$ by

$$
\mathcal{V}^{+}:=\mathcal{V}^{+}(q)=V^{+} \sqrt{\frac{1}{1+V^{+2} q^{2}}} \text { and } \mathcal{V}_{i}^{-}:=\mathcal{V}_{i}^{-}(q)=V_{i}^{-} \sqrt{\frac{1}{1+V_{i}^{-2} q^{2}}}
$$


These fictitious velocities will be helpful to turn the 3D-problem into the sum of 2D-problems indexed by the variable $q$. Note that $\mathcal{V}^{+}(0)$ and $\mathcal{V}_{i}^{-}(0)$ correspond to the real velocities $V^{+}$and $V_{i}^{-}$.

3. Definition of the functions $\kappa^{+}$and $\kappa_{i}^{-}$. For $i \in\{P f, P s, S\}$ and $\left(q_{x}, q_{y}\right) \in \mathbb{C} \times \mathbb{R}$, we define the functions

$$
\kappa^{+}:=\kappa^{+}\left(q_{x}, q_{y}\right)=\left(\frac{1}{V^{+2}}+q_{x}^{2}+q_{y}^{2}\right)^{1 / 2}=\left(\frac{1}{\mathcal{V}^{+2}\left(q_{y}\right)}+q_{x}^{2}\right)^{1 / 2} .
$$

and

$$
\kappa_{i}^{-}:=\kappa_{i}^{-}\left(q_{x}, q_{y}\right)=\left(\frac{1}{V_{i}^{-2}}+q_{x}^{2}+q_{y}^{2}\right)^{1 / 2}=\left(\frac{1}{\mathcal{V}_{i}^{-2}\left(q_{y}\right)}+q_{x}^{2}\right)^{1 / 2} .
$$

4. Definition of the reflection and transmission coefficients. For a given $\left(q_{x}, q_{y}\right) \in$ $\mathbb{C} \times \mathbb{R}$, we denote by $\mathcal{R}\left(q_{x}, q_{y}\right), \mathcal{T}_{P f}\left(q_{x}, q_{y}\right), \mathcal{T}_{P s}\left(q_{x}, q_{y}\right)$ and $\mathcal{T}_{S}\left(q_{x}, q_{y}\right)$ the solution of the linear system

$$
\mathcal{A}\left(q_{x}, q_{y}\right)\left[\begin{array}{c}
\mathcal{R}\left(q_{x}, q_{y}\right) \\
\mathcal{T}_{P f}\left(q_{x}, q_{y}\right) \\
\mathcal{T}_{P s}\left(q_{x}, q_{y}\right) \\
\mathcal{T}_{S}\left(q_{x}, q_{y}\right)
\end{array}\right]=-\frac{1}{2 \kappa^{+}\left(q_{x}, q_{y}\right) V^{+2}}\left[\begin{array}{c}
\frac{\kappa^{+}\left(q_{x}, q_{y}\right)}{\rho^{+}} \\
1 \\
0 \\
1
\end{array}\right],
$$

where the matrix $\mathcal{A}\left(q_{x}, q_{y}\right)$ is defined by:

$$
\begin{aligned}
& \mathcal{A}\left(q_{x}, q_{y}\right)= \\
& {\left[\begin{array}{cccc}
-\frac{\mathcal{\kappa}^{+}\left(q_{x}, q_{y}\right)}{\rho^{+}} & \left(\mathcal{P}_{11}+\mathcal{P}_{21}\right) \kappa_{P f}^{-}\left(q_{x}, q_{y}\right) & \left(\mathcal{P}_{12}+\mathcal{P}_{22}\right) \kappa_{P S}^{-}\left(q_{x}, q_{y}\right) & \left(1-\frac{\rho_{f}^{-}}{\rho_{w}^{-}}\right)\left(q_{x}^{2}+q_{y}^{2}\right) \\
1 & \frac{m^{-}}{V_{P f}^{-2}}\left(\beta^{-} \mathcal{P}_{11}+\mathcal{P}_{21}\right) & \frac{m^{-}}{V_{P s}^{-2}}\left(\beta^{-} \mathcal{P}_{12}+\mathcal{P}_{22}\right) & 0 \\
0 & 2 \mathcal{P}_{11} \kappa_{P f}^{-}\left(q_{x}, q_{y}\right) & 2 \mathcal{P}_{12} \kappa_{P S}^{-}\left(q_{x}, q_{y}\right) & \kappa_{S}^{-2}\left(q_{x}, q_{y}\right)+q_{x}^{2}+q_{y}^{2} \\
1 & \mathcal{A}_{4,2}\left(q_{x}, q_{y}\right) & \mathcal{A}_{4,3}\left(q_{x}, q_{y}\right) & 2 \mu^{-}\left(q_{x}^{2}+q_{y}^{2}\right) \kappa_{S}^{-}\left(q_{x}, q_{y}\right)
\end{array}\right],}
\end{aligned}
$$

with

$$
A_{4,2}\left(q_{x}, q_{y}\right)=\frac{\left(\lambda^{-}+m^{-} \beta^{-2}\right) \mathcal{P}_{11}+m^{-} \beta^{-} \mathcal{P}_{21}}{V_{P f}^{-2}}+2 \mu^{-} \kappa_{P f}^{-2}\left(q_{x}, q_{y}\right) \mathcal{P}_{11}
$$




$$
A_{4,3}\left(q_{x}, q_{y}\right)=\frac{\left(\lambda^{-}+m^{-} \beta^{-2}\right) \mathcal{P}_{12}+m^{-} \beta^{-} \mathcal{P}_{22}}{V_{P s}^{-2}}+2 \mu^{-} \kappa_{P s}^{-2}\left(q_{x}, q_{y}\right) \mathcal{P}_{12}
$$

We also denote by $V_{\max }$ the greatest velocity in the two media: $V_{\max }=\max \left(V^{+}, V_{P f}^{-}, V_{P_{S}}^{-}, V_{S}^{-}\right)$.

We can now present the expression of the solution to the Green Problem :

Theorem 3.1. The pressure and the displacement in the top medium are given by $p^{+}(x, 0, z, t)=p_{\text {inc }}^{+}(x, z, t)+\frac{d \xi_{\text {ref }}^{+}}{d t}(x, z, t)$ and $\boldsymbol{u}^{+}(x, 0, z, t)=\boldsymbol{u}_{\text {inc }}^{+}(x, z, \tau) d \tau+\boldsymbol{u}_{\text {ref }}^{+}(x, y, \tau) d \tau$, and the displacement in the bottom medium is given by

$$
\boldsymbol{u}_{s}^{-}(x, 0, z, t)=\boldsymbol{u}_{P f}^{-}(x, z, t)+\boldsymbol{u}_{P S}^{-}(x, z, t)+\boldsymbol{u}_{S}^{-}(x, z, t)
$$

where

- $p_{\text {inc }}^{+}$and $u_{\text {inc }}^{+}$are respectively the pressure and the displacement of the incident wave and satisfy:

$$
\left\{\begin{array}{l}
p_{\text {inc }}^{+}(x, z, t):=\frac{\delta\left(t-t_{0}\right)}{4 \pi V^{+2} r} \\
u_{\text {inc }, x}(x, z, t):=\frac{x t H\left(t-t_{0}\right)}{4 \pi V^{+2} r^{3} \rho^{+}} \\
u_{\text {inc }, z}(x, z, t):=\frac{(z-h) t H\left(t-t_{0}\right)}{4 \pi V^{+2} r^{3} \rho^{+}},
\end{array}\right.
$$

where $\delta$ and $H$ respectively denote the usual Dirac and Heaviside distributions. Moreover we set $r=\left(x^{2}+(z-h)^{2}\right)^{1 / 2}$ and $t_{0}=r / V^{+}$denotes the time arrival of the incident wave at point $(x, 0, z)$.

- $\xi_{\text {ref }}^{+}$and $u_{\text {ref }}^{+}$are respectively the primitive of the pressure with respect to the time and the displacement of the reflected wave and satisfy :

$$
\left\{\begin{array}{l}
\xi_{\mathrm{ref}}^{+}(x, z, t)=-\int_{0}^{q_{1}(t)} \frac{\Im m\left[\mathcal{K}^{+}(v(t, q)) \mathcal{R}(v(t, q))\right]}{\pi^{2} r \sqrt{q^{2}+q_{0}^{2}(t)}} d q, \\
u_{\mathrm{ref}, x}^{+}(x, z, t)=-\int_{0}^{q_{1}(t)} \frac{\Im m\left[\mathrm{i} v(t, q) \mathcal{K}^{+}(v(t, q)) \mathcal{R}(v(t, q))\right]}{\pi^{2} r \rho^{+} \sqrt{q^{2}+q_{0}^{2}(t)}} d q, \\
u_{\mathrm{ref}, z}^{+}(x, z, t)=-\int_{0}^{q_{1}(t)} \frac{\Im m\left[\mathcal{K}^{+2}(v(t, q)) \mathcal{R}(v(t, q))\right]}{\pi^{2} r \rho^{+} \sqrt{q^{2}+q_{0}^{2}(t)}} d q,
\end{array}\right.
$$


if $t_{\mathrm{h}_{1}}<t \leq t_{0}$ and $\frac{x}{r}>\frac{V^{+}}{V_{\max }}$,

$$
\left\{\begin{aligned}
\xi_{\mathrm{ref}}^{+}(x, z, t)= & -\int_{q_{0}(t)}^{q_{1}(t)} \frac{\Im m\left[\mathcal{K}^{+}(v(t, q)) \mathcal{R}(v(t, q))\right]}{\pi^{2} r \sqrt{q^{2}-q_{0}^{2}(t)}} d q \\
& +\int_{0}^{q_{0}(t)} \frac{\Re e\left[\mathcal{K}^{+}(\gamma(t, q)) \mathcal{R}(\gamma(t, q))\right]}{\pi^{2} r \sqrt{q_{0}^{2}(t)-q^{2}}} d q, \\
u_{\mathrm{ref}, x}^{+}(x, z, t)= & -\int_{q_{0}(t)}^{q_{1}(t)} \frac{\Im m\left[\left(\mathrm{i} v(t, q) \kappa^{+}(v(t, q)) \mathcal{R}(v(t, q))\right]\right.}{\pi^{2} r \rho^{+} \sqrt{q^{2}-q_{0}^{2}(t)}} d q \\
& +\int_{0}^{q_{0}(t)} \frac{\Re e\left[\mathrm{i} \gamma(t, q) \kappa^{+}(\gamma(t, q)) \mathcal{R}(\gamma(t, q))\right]}{\pi^{2} r \rho^{+} \sqrt{q_{0}^{2}(t)-q^{2}}} d q, \\
u_{\mathrm{ref}, y}^{+}(x, z, t)= & -\int_{q_{0}(t)}^{q_{1}(t)} \frac{\Im m\left[\mathcal{K}^{+2}(v(t, q)) \mathcal{R}(v(t, q))\right]}{\pi^{2} r \rho^{+} \sqrt{q^{2}-q_{0}^{2}(t)}} d q \\
& +\int_{0}^{q_{0}(t)} \frac{\Re e\left[\kappa^{+2}(\gamma(t)) \mathcal{R}(\gamma(t))\right]}{\pi^{2} r \rho^{+} \sqrt{q_{0}^{2}(t)-q^{2}}} d q,
\end{aligned}\right.
$$

if $t_{0}<t \leq t_{\mathrm{h}_{2}}$ and $\frac{x}{r}>\frac{V^{+}}{V_{\max }}$,

$$
\left\{\begin{array}{l}
\xi_{\text {ref }}^{+}(x, z, t)=\int_{0}^{q_{0}(t)} \frac{\Re e\left[\mathcal{K}^{+}(\gamma(t, q)) \mathcal{R}(\gamma(t, q))\right]}{\pi^{2} r \sqrt{q_{0}^{2}(t)-q^{2}}} d q, \\
u_{\mathrm{ref}, x}^{+}(x, z, t)=\int_{0}^{q_{0}(t)} \frac{\Re e\left[\mathrm{i} \gamma(t, q) \mathcal{K}^{+}(\gamma(t, q)) \mathcal{R}(\gamma(t, q))\right]}{2 \pi^{2} r \rho^{+} \sqrt{q_{0}^{2}(t)-q^{2}}} d q, \\
u_{\mathrm{ref}, y}^{+}(x, z, t)=\int_{0}^{q_{0}(t)} \frac{\Re e\left[\mathcal{K}^{+2}(\gamma(t)) \mathcal{R}(\gamma(t))\right]}{\pi^{2} r \rho^{+} \sqrt{q_{0}^{2}(t)-q^{2}}} d q,
\end{array}\right.
$$

if $t_{\mathrm{h}_{2}}<t$ and $\frac{x}{r}>\frac{V^{+}}{V_{\max }}$ or if $t_{0}<t$ and $\frac{x}{r} \leq \frac{V^{+}}{V_{\max }}$ and

$$
\xi_{\text {ref }}(x, y, t)=0 \text { and } u_{\text {ref }}(x, y, t)=0 \text { else } .
$$

We set here $r=\left(x^{2}+(z+h)^{2}\right)^{1 / 2}$ and $t_{0}=r / V^{+}$denotes the arrival time of the re- 
flected body wave at point $(x, 0, z)$,

$$
t_{h_{1}}=(z+h) \sqrt{\frac{1}{V^{+2}}-\frac{1}{V_{\max }^{2}}}+\frac{|x|}{V_{\max }}
$$

denotes the arrival time of the reflected head-wave at point $(x, 0, z)$ and

$$
t_{h_{2}}=\frac{r}{z+h} \sqrt{\frac{1}{V^{+2}}-\frac{1}{V_{\max }^{2}}}
$$

denotes the time after which there is no longer head wave at point $(x, 0, z)$, (contrary to the $2 \mathrm{D}$ case, this time does not coincide with the arrival time of the body wave). We also define the functions $\gamma, v, q_{0}$ and $q_{1}$ by

$$
\begin{gathered}
\gamma:\left\{t \in \mathbb{R} \mid t>t_{0}\right\} \times \mathbb{R} \mapsto \mathbb{C}:=\gamma\left(t, q_{y}\right)=\mathrm{i} \frac{x t}{r^{2}}+\frac{z+h}{r} \sqrt{\frac{t^{2}}{r^{2}}-\frac{1}{\mathcal{V}^{+2}\left(q_{y}\right)}} \\
v:\left\{t \in \mathbb{R} \mid t_{h_{1}}<t<t_{h_{2}}\right\} \times \mathbb{R} \mapsto \mathbb{C}:=v\left(t, q_{y}\right)=-\mathrm{i}\left(\frac{z+h}{r}-\sqrt{\left.\frac{1}{\mathcal{V}^{+2}\left(q_{y}\right)}-\frac{t^{2}}{r^{2}}+\frac{x}{r^{2}} t\right),}\right. \\
q_{0}: \mathbb{R} \rightarrow \mathbb{R}:=q_{0}(t)=\sqrt{\left|\frac{t^{2}}{r^{2}}-\frac{1}{V^{+2}}\right|}
\end{gathered}
$$

and

$$
q_{1}: \mathbb{R} \rightarrow \mathbb{R}:=q_{1}(t)=\sqrt{\frac{1}{x^{2}}\left(t-(z+h) \sqrt{\frac{1}{V^{+2}}-\frac{1}{V_{\max }^{2}}}\right)^{2}-\frac{1}{V_{\max }^{2}}} .
$$

Remark 3.1. For the practical computation of the pressure, we won't have to explicitly compute the derivative of the function $\xi_{\text {ref }}^{+}$(which would be rather tedious), since

$$
p_{\text {ref }}^{+} * f=\partial_{t} \xi_{\text {ref }}^{+} * f=\xi_{\text {ref }}^{+} * f^{\prime} .
$$

Therefore, we'll only have to compute the derivative of the source function $f$.

- $\boldsymbol{u}_{P f}^{-}(x, z, t)$ is the displacement of the transmitted $P f$ wave and satisfies:

$$
\left\{\begin{array}{l}
u_{P f, x}^{-}(x, z, t)=-\frac{\mathcal{P}_{11}}{\pi^{2}} \int_{0}^{q_{1}(t)} \Re e\left[\mathrm{i} v(t, q) \mathcal{T}_{P f}(v(t, q)) \frac{\partial v}{\partial t}(t, q)\right] d q, \\
u_{P f, z}^{-}(x, z, t)=\frac{\mathcal{P}_{11}}{\pi^{2}} \int_{0}^{q_{1}(t)} \Re e\left[\kappa_{P f}^{-}(v(t, q)) \mathcal{T}_{P f}(v(t, q)) \frac{\partial v}{\partial t}(t, q)\right] d q,
\end{array}\right.
$$


if $t_{h_{1}}<t \leq t_{0}$ and $\left|\Im m\left[\gamma\left(t_{0}, 0\right)\right]\right|>\frac{1}{V_{\max }}$,

$$
\left\{\begin{aligned}
u_{P f, x}^{-}(x, z, t)= & -\frac{\mathcal{P}_{11}}{\pi^{2}} \int_{0}^{q_{0}(t)} \Re e\left[\mathrm{i} \gamma(t, q) \mathcal{T}_{P f}(\gamma(t, q)) \frac{\partial \gamma}{\partial t}(t, q)\right] d q \\
& -\frac{\mathcal{P}_{11}}{\pi^{2}} \int_{q_{0}(t)}^{q_{1}(t)} \Re e\left[\mathrm{i} v(t, q) \mathcal{T}_{P f}(v(t, q)) \frac{\partial v}{\partial t}(t, q)\right] d q, \\
u_{P f, z}^{-}(x, z, t)= & \frac{\mathcal{P}_{11}}{\pi^{2}} \int_{0}^{q_{0}(t)} \Re e\left[\kappa_{P f}^{-}(\gamma(t, q)) \mathcal{T}_{P f}(\gamma(t, q)) \frac{\partial \gamma}{\partial t}(t, q)\right] d q \\
& +\frac{\mathcal{P}_{11}}{\pi^{2}} \int_{q_{0}(t)}^{q_{1}(t)} \Re e\left[\kappa_{P f}^{-}(v(t, q)) \mathcal{T}_{P f}(v(t, q)) \frac{\partial v}{\partial t}(t, q)\right] d q,
\end{aligned}\right.
$$

if $t_{0}<t \leq t_{h_{2}}$ and $\left|\Im m\left[\gamma\left(t_{0}, 0\right)\right]\right|>\frac{1}{V_{\max }}$,

$$
\left\{\begin{array}{l}
u_{P f, x}^{-}(x, z, t)=-\frac{\mathcal{P}_{11}}{\pi^{2}} \int_{0}^{q_{0}(t)} \Re e\left[\mathrm{i} \gamma(t, q) \mathcal{T}_{P f}(\gamma(t, q)) \frac{\partial \gamma}{\partial t}(t, q)\right] d q, \\
u_{P f, z}^{-}(x, z, t)=\frac{\mathcal{P}_{11}}{\pi^{2}} \int_{0}^{q_{0}(t)} \Re e\left[\mathcal{K}_{P f}^{-}(\gamma(t, q)) \mathcal{T}_{P f}(\gamma(t, q)) \frac{\partial \gamma}{\partial t}(t, q)\right] d q,
\end{array}\right.
$$

if $t_{h_{2}}<t$ and $\left|\Im m\left[\gamma\left(t_{0}, 0\right)\right]\right|>\frac{1}{V_{\max }}$ or if $t_{0}<t$ and $\left|\Im m\left[\gamma\left(t_{0}, 0\right)\right]\right| \leq \frac{1}{V_{\max }}$ and $\boldsymbol{u}_{P f}^{-}(x, z, t)=0$ else.

$t_{0}$ denotes here the arrival time of the $P f$ body wave at point $(x, 0, z)$ (we recall in appendix the computation of $t_{0}$ ),

$$
t_{h_{1}}=h \sqrt{\frac{1}{V^{+2}}-\frac{1}{V_{\max }^{2}}}-z \sqrt{\frac{1}{V_{P f}^{-2}}-\frac{1}{V_{\max }^{2}}}+\frac{|x|}{V_{\max }}
$$

denotes the arrival time of the $P f$ head wave at point $(x, 0, z)$,

$$
t_{h_{2}}=\frac{h^{2}+z^{2}-h z\left(\frac{c_{2}}{c_{1}}+\frac{c_{1}}{c_{2}}\right)+x^{2}}{\frac{h}{c_{1}}-\frac{z}{c_{2}}}
$$

denotes the time after which there is no longer head wave at point $(x, 0, z)$, where

$$
c_{1}=\sqrt{\frac{1}{V^{+2}}-\frac{1}{V_{\max }^{2}}} \text { and } c_{2}=\sqrt{\frac{1}{V_{P f}^{2}}-\frac{1}{V_{\max }^{2}}} .
$$


The function $q_{0}:\left[t_{0} ;+\infty\right] \mapsto \mathbb{R}^{+}$is the reciprocal function of $\tilde{t}_{0}: \mathbb{R}^{+} \mapsto:\left[t_{0},+\infty\right]$, where $\widetilde{t}_{0}(q)$ is the arrival time at point $(x, 0, z)$ of the fictitious $P f$ body wave, propagating at a velocity $\mathcal{V}^{+}(q)$ in the top layer and at velocity $\mathcal{V}_{P f}^{-}(q)$ in the bottom layer (we recall in appendix the computation of $\left.\widetilde{t}_{0}(q)\right)$.

The function $q_{1}:\left[t_{1} ; t_{0}\right] \mapsto \mathbb{R}^{+}$is defined by

$$
q_{1}(t)=\sqrt{\frac{1}{x^{2}}\left(t+z \sqrt{\frac{1}{V_{P f}^{-2}}-\frac{1}{V_{\max }^{2}}}-h \sqrt{\frac{1}{V^{+2}}-\frac{1}{V_{\max }^{2}}}\right)^{2}-\frac{1}{V_{\max }^{2}} .}
$$

The function $\gamma:\left\{(t, q) \in \mathbb{R}^{+} \times \mathbb{R}^{+} \mid t>\tilde{t}_{0}(q)\right\} \mapsto \mathbb{C}$ is implicitly defined as the only root of the function

$$
\mathcal{F}(\gamma, q, t)=-z\left(\frac{1}{\mathcal{V}_{P f}^{-2}(q)}+\gamma^{2}\right)^{1 / 2}+h\left(\frac{1}{\mathcal{V}^{+2}(q)}+\gamma^{2}\right)^{1 / 2}+i \gamma x-t
$$

whose real part is positive.

The function $v: E_{1} \cup E_{2} \mapsto \mathbb{C}$ is implicitly defined as the only root of the function

$$
\mathcal{F}(v, q, t)=-z\left(\frac{1}{\mathcal{V}_{P f}^{-2}(q)}+v^{2}\right)^{1 / 2}+h\left(\frac{1}{\mathcal{V}^{+2}(q)}+v^{2}\right)^{1 / 2}+i v x-t
$$

such that $\Im m\left[\partial_{t} v(t, q)\right]<0$, with

$$
E_{1}=\left\{(t, q) \in \mathbb{R}^{+} \times \mathbb{R}^{+} \mid t_{h_{1}}<t<t_{0} \text { and } 0<q<q_{0}(t)\right\}
$$

and

$$
E_{2}=\left\{(t, q) \in \mathbb{R}^{+} \times \mathbb{R}^{+} \mid t_{0}<t<t_{h_{1}} \text { and } q_{0}(t)<q<q_{1}(t)\right\}
$$

- $\boldsymbol{u}_{P S}^{-}(x, z, t)$ is the displacement of the transmitted $P S$ wave and satisfies:

$$
\begin{aligned}
& \left\{\begin{array}{l}
u_{P_{s, x}}^{-}(x, z, t)=-\frac{\mathcal{P}_{12}}{\pi^{2}} \int_{0}^{q_{1}(t)} \Re e\left[\mathrm{i} v(t, q) \mathcal{T}_{P_{s}}(v(t, q)) \frac{d v}{d t}(t, q)\right] d q, \\
u_{P_{s, z}}^{-}(x, z, t)=\frac{\mathcal{P}_{12}}{\pi^{2}} \int_{0}^{q_{1}(t)} \Re e\left[\kappa_{P_{s}}^{-}(v(t), q) \mathcal{T}_{P_{s}}(v(t, q)) \frac{d v}{d t}(t, q)\right] d q,
\end{array}\right. \\
& \text { if } t_{h_{1}}<t \leq t_{0} \text { and }\left|\Im m\left[\gamma\left(t_{0}, 0\right)\right]\right|>\frac{1}{V_{\max }},
\end{aligned}
$$




$$
\left\{\begin{aligned}
u_{P s, x}^{-}(x, z, t)= & -\frac{\mathcal{P}_{12}}{\pi^{2}} \int_{0}^{q_{0}(t)} \Re e\left[\mathrm{i} \gamma(t, q) \mathcal{T}_{P f}(\gamma(t, q)) \frac{\partial \gamma}{\partial t}(t, q)\right] d q \\
& -\frac{\mathcal{P}_{12}}{\pi^{2}} \int_{q_{0}(t)}^{q_{1}(t)} \Re e\left[\mathrm{i} v(t, q) \mathcal{T}_{P s}(v(t, q)) \frac{d v}{d t}(t, q)\right] d q \\
u_{P s, z}^{-}(x, z, t)= & \frac{\mathcal{P}_{12}}{\pi^{2}} \int_{0}^{q_{0}(t)} \Re e\left[\kappa_{P s}^{-}(\gamma(t, q)) \mathcal{T}_{P s}(\gamma(t, q)) \frac{d \gamma}{d t}(t, q)\right] d q \\
& +\frac{\mathcal{P}_{12}}{\pi^{2}} \int_{q_{0}(t)}^{q_{1}(t)} \Re e\left[\kappa_{P s}^{-}(v(t), q) \mathcal{T}_{P_{s}}(v(t, q)) \frac{d v}{d t}(t, q)\right] d q,
\end{aligned}\right.
$$

if $t_{0}<t \leq t_{h_{2}}$ and $\left|\Im m\left[\gamma\left(t_{0}, 0\right)\right]\right|>\frac{1}{V_{\max }}$,

$$
\left\{\begin{array}{l}
u_{P_{s, x}}^{-}(x, z, t)=-\frac{\mathcal{P}_{12}}{\pi^{2}} \int_{0}^{q_{0}(t)} \Re e\left[\mathrm{i} \gamma(t, q) \mathcal{T}_{P_{s}}(\gamma(t, q)) \frac{d \gamma}{d t}(t, q)\right] d q, \\
u_{P_{s, z}}^{-}(x, z, t)=\frac{\mathcal{P}_{12}}{\pi^{2}} \int_{0}^{q_{0}(t)} \Re e\left[\kappa_{P s}^{-}(\gamma(t, q)) \mathcal{T}_{P_{s}}(\gamma(t, q)) \frac{d \gamma}{d t}(t, q)\right] d q,
\end{array}\right.
$$

if $t_{h_{2}}<t$ and $\left|\Im m\left[\gamma\left(t_{0}, 0\right)\right]\right|>\frac{1}{V_{\max }}$ or if $t_{0}<t$ and $\left|\Im m\left[\gamma\left(t_{0}, 0\right)\right]\right| \leq \frac{1}{V_{\max }}$ and $\boldsymbol{u}_{P_{S}}^{-}(x, z, t)=0$ else. $t_{0}$ denotes here the arrival time of the $P_{s}$ body wave at point $(x, 0, z)$,

$$
t_{h_{1}}=h \sqrt{\frac{1}{V^{+2}}-\frac{1}{V_{\max }^{2}}}-z \sqrt{\frac{1}{V_{P s}^{-2}}-\frac{1}{V_{\max }^{2}}}+\frac{|x|}{V_{\max }}
$$

denotes the arrival time of the $P_{S}$ head wave at point $(x, 0, z)$ and

$$
t_{h_{2}}=\frac{h^{2}+z^{2}-h z\left(\frac{c_{2}}{c_{1}}+\frac{c_{1}}{c_{2}}\right)+x^{2}}{\frac{h}{c_{1}}-\frac{z}{c_{2}}}
$$

denotes the time after which there is no longer head wave at point $(x, 0, z)$, where

$$
c_{1}=\sqrt{\frac{1}{V^{+2}}-\frac{1}{V_{\max }^{2}}} \text { and } c_{2}=\sqrt{\frac{1}{V_{P_{S}}^{2}}-\frac{1}{V_{\max }^{2}}} .
$$

The function $q_{0}:\left[t_{0} ;+\infty\right] \mapsto \mathbb{R}^{+}$is the reciprocal function of $\tilde{t}_{0}: \mathbb{R}^{+} \mapsto:\left[t_{0},+\infty\right]$, where $\widetilde{t}_{0}(q)$ is the arrival time at point $(x, 0, z)$ of the fictitious $P_{S}$ body wave, propagating at a velocity $\mathcal{V}^{+}(q)$ in the top layer and at velocity $\mathcal{V}_{P_{S}}^{-}(q)$ in the bottom layer. 
The function $q_{1}:\left[t_{1} ; t_{0}\right] \mapsto \mathbb{R}^{+}$is defined by

$$
q_{1}(t)=\sqrt{\frac{1}{x^{2}}\left(t+z \sqrt{\frac{1}{V_{P_{s}}^{-2}}-\frac{1}{V_{\max }^{2}}}-h \sqrt{\frac{1}{V^{+2}}-\frac{1}{V_{\max }^{2}}}\right)^{2}-\frac{1}{V_{\max }^{2}} .}
$$

The function $\gamma:\left\{(t, q) \in \mathbb{R}^{+} \times \mathbb{R}^{+} \mid t>\tilde{t}_{0}(q)\right\} \mapsto \mathbb{C}$ is implicitly defined as the only root of the function

$$
\mathcal{F}(\gamma, q, t)=-z\left(\frac{1}{\mathcal{V}_{P_{s}}^{-2}(q)}+\gamma^{2}\right)^{1 / 2}+h\left(\frac{1}{\mathcal{V}^{+2}(q)}+\gamma^{2}\right)^{1 / 2}+i \gamma x-t
$$

whose real part is positive.

The function $v: E_{1} \cup E_{2} \mapsto \mathbb{C}$ is implicitly defined as the only root of the function

$$
\mathcal{F}(v, q, t)=-z\left(\frac{1}{\mathcal{V}_{P s}^{-2}(q)}+v^{2}\right)^{1 / 2}+h\left(\frac{1}{\mathcal{V}+2(q)}+v^{2}\right)^{1 / 2}+i v x-t
$$

such that $\Im m\left[\partial_{t} v(t, q)\right]<0$, with

$$
E_{1}=\left\{(t, q) \in \mathbb{R}^{+} \times \mathbb{R}^{+} \mid t_{h_{1}}<t<t_{0} \text { and } 0<q<q_{0}(t)\right\}
$$

and

$$
E_{2}=\left\{(t, q) \in \mathbb{R}^{+} \times \mathbb{R}^{+} \mid t_{0}<t<t_{h_{1}} \text { and } q_{0}(t)<q<q_{1}(t)\right\} .
$$

- $\boldsymbol{u}_{S}^{-}(x, z, t)$ is the displacement of the transmitted $S$ wave and satisfies:

$$
\begin{gathered}
\left\{\begin{array}{c}
u_{S, x}^{-}(x, z, t)=-\frac{1}{\pi^{2}} \int_{0}^{q_{1}(t)} \Re e\left[\mathrm{i} v(t, q) \kappa_{S}^{-}(v(t, q)) \mathcal{T}_{S}(v(t, q)) \frac{d v}{d t}(t, q)\right] d q, \\
u_{S, z}^{-}(x, z, t)=\frac{1}{\pi^{2}} \int_{0}^{q_{1}(t)} \Re e\left[\left(v^{2}(t, q)+q^{2}\right) \mathcal{T}_{S}(v(t, q)) \frac{d v}{d t}(t, q)\right] d q,
\end{array}\right. \\
\text { if } t_{h_{1}}<t \leq t_{0} \text { and }\left|\Im m\left[\gamma\left(t_{0}, 0\right)\right]\right|>\frac{1}{V_{\max }}, \\
\left\{\begin{aligned}
& u_{S, x}^{-}(x, z, t)=-\frac{1}{\pi^{2}} \int_{0}^{q_{0}(t)} \Re e\left[\mathrm{i} \gamma(t, q) \mathcal{K}_{S}^{-}(\gamma(t, q)) \mathcal{T}_{S}(\gamma(t, q)) \frac{d \gamma}{d t}(t, q)\right] d q, \\
& u_{S, z}^{-}(x, z, t)= \frac{1}{\pi^{2}} \int_{q_{0}(t)}^{q_{1}(t)} \Re e\left[\mathrm{i} v(t, q) \mathcal{K}_{S}^{-}(v(t, q)) \mathcal{T}_{S}(v(t, q)) \frac{d v}{d t}(t, q)\right] d q \\
&+\frac{1}{\pi^{2}} \int_{0}^{q_{0}(t)} \Re e\left[\left(\gamma^{2}(t, q)+q^{2}\right) \mathcal{T}_{S}(\gamma(t, q)) \frac{d \gamma}{d t}(t, q)\right] d q \\
& \pi^{2} \int_{q_{0}(t)}^{q_{1}(t)} \Re e\left[\left(v^{2}(t, q)+q^{2}\right) \mathcal{T}_{S}(v(t, q)) \frac{d v}{d t}(t, q)\right] d q,
\end{aligned}\right.
\end{gathered}
$$


if $t_{0}<t \leq t_{h_{2}}$ and $\left|\Im m\left[\gamma\left(t_{0}, 0\right)\right]\right|>\frac{1}{V_{\max }}$,

$$
\left\{\begin{array}{l}
u_{S, x}^{-}(x, z, t)=-\frac{1}{\pi^{2}} \int_{0}^{q_{0}(t)} \Re e\left[\mathrm{i} \gamma(t, q) \kappa_{S}^{-}(\gamma(t, q)) \mathcal{T}_{S}(\gamma(t, q)) \frac{d \gamma}{d t}(t, q)\right] d q, \\
u_{S, z}^{-}(x, z, t)=\frac{1}{\pi^{2}} \int_{0}^{q_{0}(t)} \Re e\left[\left(\gamma^{2}(t, q)+q^{2}\right) \mathcal{T}_{S}(\gamma(t, q)) \frac{d \gamma}{d t}(t, q)\right] d q
\end{array}\right.
$$

if $t_{h_{2}}<t$ and $\left|\Im m\left[\gamma\left(t_{0}, 0\right)\right]\right|>\frac{1}{V_{\max }}$ or if $t_{0}<t$ and $\left|\Im m\left[\gamma\left(t_{0}, 0\right)\right]\right| \leq \frac{1}{V_{\max }}$ and $\boldsymbol{u}_{P_{S}}^{-}(x, z, t)=0$ else. $t_{0}$ denotes here the arrival time of the $S$ body wave at point $(x, 0, z)$ (we recall in appendix the computation of $\left.t_{0}\right)$,

$$
t_{h_{1}}=h \sqrt{\frac{1}{V^{+2}}-\frac{1}{V_{\max }^{2}}}-z \sqrt{\frac{1}{V_{S}^{-2}}-\frac{1}{V_{\max }^{2}}}+\frac{|x|}{V_{\max }}
$$

denotes the arrival time of the $S$ head-wave at point $(x, 0, z)$ and

$$
t_{h_{2}}=\frac{h^{2}+z^{2}-h z\left(\frac{c_{2}}{c_{1}}+\frac{c_{1}}{c_{2}}\right)+x^{2}}{\frac{h}{c_{1}}-\frac{z}{c_{2}}}
$$

denotes the time after which there is no longer head wave at point $(x, 0, z)$, where

$$
c_{1}=\sqrt{\frac{1}{V^{+2}}-\frac{1}{V_{\max }^{2}}} \text { and } c_{2}=\sqrt{\frac{1}{V_{S}^{2}}-\frac{1}{V_{\max }^{2}}} .
$$

The function $q_{0}:\left[t_{0} ;+\infty\right] \mapsto \mathbb{R}^{+}$is the reciprocal function of $\tilde{t}_{0}: \mathbb{R}^{+} \mapsto:\left[t_{0},+\infty\right]$, where $\widetilde{t}_{0}(q)$ is the arrival time at point $(x, 0, z)$ of the fictitious $S$ body wave, propagating at a velocity $\mathcal{V}^{+}(q)$ in the top layer and at velocity $\mathcal{V}_{S}^{-}(q)$ in the bottom layer (we recall in appendix the computation of $\widetilde{t}_{0}(q)$ ).

The function $q_{1}:\left[t_{1} ; t_{0}\right] \mapsto \mathbb{R}^{+}$is defined by

$$
q_{1}(t)=\sqrt{\frac{1}{x^{2}}\left(t+z \sqrt{\frac{1}{V_{S}^{-2}}-\frac{1}{V_{\max }^{2}}}-h \sqrt{\frac{1}{V^{+2}}-\frac{1}{V_{\max }^{2}}}\right)^{2}-\frac{1}{V_{\max }^{2}} .}
$$

The function $\gamma:\left\{(t, q) \in \mathbb{R}^{+} \times \mathbb{R}^{+} \mid t>\tilde{t}_{0}(q)\right\} \mapsto \mathbb{C}$ is implicitly defined as the only root of the function

$$
\mathcal{F}(\gamma, q, t)=-z\left(\frac{1}{\mathcal{V}_{S}^{-2}(q)}+\gamma^{2}\right)^{1 / 2}+h\left(\frac{1}{\mathcal{V}^{+2}(q)}+\gamma^{2}\right)^{1 / 2}+i \gamma x-t
$$


whose real part is positive.

The function $v: E_{1} \cup E_{2} \mapsto \mathbb{C}$ is implicitly defined as the only root of the function

$$
\mathcal{F}(v, q, t)=-z\left(\frac{1}{\mathcal{V}_{S}^{-2}(q)}+v^{2}\right)^{1 / 2}+h\left(\frac{1}{\mathcal{V}^{+2}(q)}+v^{2}\right)^{1 / 2}+i v x-t
$$

such that $\Im m\left[\partial_{t} v(t, q)\right]<0$, with

$$
E_{1}=\left\{(t, q) \in \mathbb{R}^{+} \times \mathbb{R}^{+} \mid t_{h_{1}}<t<t_{0} \text { and } 0<q<q_{0}(t)\right\}
$$

and

$$
E_{2}=\left\{(t, q) \in \mathbb{R}^{+} \times \mathbb{R}^{+} \mid t_{0}<t<t_{h_{1}} \text { and } q_{0}(t)<q<q_{1}(t)\right\}
$$

\section{Proof of the theorem}

To prove the theorem, we use the Cagniard-de Hoop method (see [1, 2, 13-15]), which consists of three steps:

1. We apply a Laplace transform in time,

$$
\tilde{u}(x, y, z, s)=\int_{0}^{+\infty} u(x, y, z, t) e^{-s t} d t
$$

and a Fourier transform in the $x$ and $y$ variables,

$$
\hat{u}\left(k_{x}, k_{y}, z, s\right)=\int_{-\infty}^{+\infty} \int_{-\infty}^{+\infty} \tilde{u}(x, y, s) e^{i\left(k_{x} x+k_{y} y\right)} d x d y
$$

to (2.17) in order to obtain an ordinary differential system whose solution $\hat{\mathcal{G}}\left(k_{x}, k_{y}, z, s\right)$ can be explicitly computed $(\S 4.1)$;

2. we apply an inverse Fourier transform in the $x$ and $y$ variables to $\mathcal{G}$ (we recall that we only need the solution at $y=0$ :

$$
\tilde{\mathcal{G}}(x, 0, z, s)=\frac{1}{4 \pi^{2}} \int_{-\infty}^{+\infty} \int_{-\infty}^{+\infty} \hat{\mathcal{G}}\left(k_{x}, k_{y}, z, s\right) e^{-\mathrm{i} k_{x} x} d k_{x} d k_{y} .
$$

And, using tools of complex analysis, we turn the inverse Fourier transform in the $x$ variable into the Laplace transform of some function $\mathcal{H}\left(x, k_{y}, z, t\right)(\S 4.2)$ :

$$
\tilde{\mathcal{G}}(x, 0, z, s)=\frac{1}{4 \pi^{2}} \int_{-\infty}^{+\infty} \int_{0}^{+\infty} \mathcal{H}\left(x, k_{y}, z, t\right) e^{-s t} d t d k_{y}
$$


3. the last step of the method consists in inverting the order of integration in (4.1) to obtain

$$
\tilde{\mathcal{G}}(x, 0, z, s)=\frac{1}{4 \pi^{2}} \int_{0}^{+\infty}\left(\int_{-q(t)}^{+q(t)} \mathcal{H}\left(x, k_{y}, z, t\right) d k_{y}\right) e^{-s t} d t .
$$

Then, using the injectivity of the Laplace transform, we identify $\mathcal{G}(x, 0, z, t)$ to

$$
\frac{1}{4 \pi^{2}} \int_{-q(t)}^{+q(t)} \mathcal{H}\left(x, k_{y}, z, t\right) d k_{y}
$$

(see $\S 4.3)$.

\subsection{The solution in the Laplace-Fourier plane}

Let us first apply a Laplace transform in time and a Fourier transform in the $x$ and $y$ variables to (2.17) to obtain

$$
\begin{cases}\left(\frac{s^{2}}{V^{+2}}+k_{x}^{2}+k_{y}^{2}\right) \hat{p}^{+}-\frac{\partial^{2} \hat{p}^{+}}{\partial z^{2}}=\frac{\delta(z-h)}{V^{+2}}, & y>0 \\ \left(\frac{s^{2}}{V_{i}^{-2}}+k_{x}^{2}+k_{y}^{2}\right) \hat{\Phi}_{i}^{-}-\frac{\partial^{2} \hat{\Phi}_{i}^{-}}{\partial z^{2}}=0, i \in\{P f, P s, S\} & y<0 \\ \hat{\mathcal{B}}\left(\hat{p}^{+}, \hat{\Phi}_{P f}^{-}, \hat{\Phi}_{P f}^{-}, \hat{\Phi}_{S}^{-}\right)=0 & y=0,\end{cases}
$$

where $\hat{\mathcal{B}}$ is the Laplace-Fourier transform of the operator $\mathcal{B}$.

From the two first equations of (4.2), we deduce that the solution $\left(\hat{p}^{+},\left(\hat{\Phi}_{i}^{-}\right)_{i \in\{P f, P s, S\}}\right)$ is such that

$$
\left\{\begin{array}{l}
\hat{p}^{+}=\hat{p}_{\text {inc }}^{+}+\hat{p}_{\text {ref }^{\prime}}^{+} \\
\hat{p}_{\text {inc }}^{+}=\frac{1}{s V^{+2} \kappa^{+}\left(\frac{k_{x}}{s}, \frac{k_{y}}{s}\right)} e^{-s|z-h| \kappa^{+}\left(\frac{k_{x}}{s}, \frac{k_{y}}{s}\right)}, \hat{p}_{\text {ref }}^{+}=R\left(k_{x}, k_{y}, s\right) e^{-s z \mathcal{K}^{+}\left(\frac{k_{x}}{s}, \frac{k_{y}}{s}\right)}, \\
\hat{\Phi}_{i}^{-}=T_{i}\left(k_{x}, k_{y}, s\right) e^{-s\left(z \kappa_{i}^{-}\left(\frac{k_{x}}{s} \frac{k_{y}}{s}\right)\right)}, \quad i \in\{P f, P s, S\}
\end{array}\right.
$$

where the coefficients $R$ and $T_{i}$ are computed by using the last equation of (4.2):

$$
\hat{\mathcal{B}}\left(\hat{p}_{\text {ref }^{\prime}}^{+} \hat{\Phi}_{P f}^{-}, \hat{\Phi}_{P_{S}}^{-}, \hat{\Phi}_{S}^{-}\right)=-\hat{\mathcal{B}}\left(\hat{p}_{\text {inc }^{\prime}}^{+}, 0,0,0\right),
$$

or, from $(4.3)$ :

$$
\hat{\mathcal{B}}\left[R\left(k_{x}, k_{y}, s\right), T_{P f}\left(k_{x}, k_{y}, s\right), T_{P s}\left(k_{x}, k_{y}, s\right), T_{S}\left(k_{x}, k_{y}, s\right)\right]=-\hat{\mathcal{B}}\left(\frac{e^{-s h \kappa^{+}\left(\frac{k_{x}}{s}, \frac{k_{y}}{s}\right)}}{s \kappa^{+}\left(\frac{k_{x}}{s}, \frac{k_{y}}{s}\right)}, 0,0,0\right) .
$$


After some calculations that we don't detail here, we obtain that $R\left(k_{x}, k_{y}, s\right), T_{P f}\left(k_{x}, k_{y}, s\right)$, $T_{P s}\left(k_{x}, k_{y}, s\right)$, and $\left.T_{S}\left(k_{x}, k_{y}, s\right)\right)$ are solution to

$$
\mathcal{A}\left(\frac{k_{x}}{s}, \frac{k_{y}}{s}\right)\left[\begin{array}{c}
R\left(k_{x}, k_{y}, s\right) \\
s^{2} T_{P f}\left(k_{x}, k_{y}, s\right) \\
s^{2} T_{P s}\left(k_{x}, k_{y}, s\right) \\
s^{3} T_{S}\left(k_{x}, k_{y}, s\right)
\end{array}\right]=-\frac{e^{-s h \kappa^{+}\left(\frac{k_{x}}{s}, \frac{k_{y}}{s}\right)}}{2 s \kappa^{+}\left(\frac{k_{x}}{s}, \frac{k_{y}}{s}\right) V^{+2}}\left[\begin{array}{c}
\frac{\kappa^{+}\left(\frac{k_{x}}{s}, \frac{k_{y}}{s}\right)}{\rho^{+}} \\
1 \\
0 \\
1
\end{array}\right] .
$$

From the definition of the reflection and transmission coefficients we deduce that

$$
\left[\begin{array}{c}
R\left(k_{x}, k_{y}, s\right) \\
s^{2} T_{P f}\left(k_{x}, k_{y}, s\right) \\
s^{2} T_{P s}\left(k_{x}, k_{y}, s\right) \\
s^{3} T_{S}\left(k_{x}, k_{y}, s\right)
\end{array}\right]=\frac{1}{s}\left[\begin{array}{c}
\mathcal{R}\left(\frac{k_{x}}{s}, \frac{k_{y}}{s}\right) \\
\mathcal{T}_{P f}\left(\frac{k_{x}}{s}, \frac{k_{y}}{s}\right) \\
\mathcal{T}_{P s}\left(\frac{k_{x}}{s}, \frac{k_{y}}{s}\right) \\
\mathcal{T}_{S}\left(\frac{k_{x}}{s}, \frac{k_{y}}{s}\right)
\end{array}\right] e^{-s h \kappa^{+}\left(\frac{k_{x}}{s}\right)} .
$$

Finally, we obtain:

$$
\left\{\begin{array}{l}
\hat{p}^{+}=\hat{p}_{\text {inc }}^{+}+\hat{p}_{\text {ref }^{\prime}}^{+} \\
\hat{p}_{\text {inc }}^{+}=\frac{1}{s V^{+2} \kappa^{+}\left(\frac{k_{x}}{s}, \frac{k_{y}}{s}\right)} e^{-s|y-h| \kappa^{+}\left(\frac{k_{x}}{s}, \frac{k_{y}}{s}\right)}, \hat{p}_{\text {ref }}^{+}=\frac{1}{s} \mathcal{R}\left(\frac{k_{x}}{s}, \frac{k_{y}}{s}\right) e^{-s(z+h) \kappa^{+}\left(\frac{k_{x}}{s}, \frac{k_{y}}{s}\right)} \\
\hat{\Phi}_{i}^{-}=\frac{1}{s^{3}} \mathcal{T}_{i}\left(\frac{k_{x}}{s}, \frac{k_{y}}{s}\right) e^{-s\left(z \kappa_{i}^{-}\left(\frac{k_{x}}{s}, \frac{k_{y}}{s}\right)-h \kappa^{+}\left(\frac{k_{x}}{s}, \frac{k_{y}}{s}\right)\right)}, \quad i \in\{P f, P s\} \\
\hat{\Phi}_{S}^{-}=\frac{1}{s^{4}} \mathcal{T}_{S}\left(\frac{k_{x}}{s}, \frac{k_{y}}{s}\right) e^{-s\left(z \kappa_{S}^{-}\left(\frac{k_{x}}{s}, \frac{k_{y}}{s}\right)-h \kappa^{+}\left(\frac{k_{x}}{s}, \frac{k_{y}}{s}\right)\right)}
\end{array}\right.
$$


and

$$
\left\{\begin{array}{l}
\hat{u}^{+}=\hat{u}_{\text {inc }}^{+}+\hat{u}_{\text {ref }}^{+} \\
\hat{u}_{\text {inc }, x}^{+}=\mathrm{i} \frac{k_{x}}{\rho^{+} s^{2}} \hat{p}_{\text {inc }}^{+}, \hat{u}_{\text {inc }, z}^{+}=\operatorname{sign}(h-z) \frac{\mathcal{K}^{+}\left(\frac{k_{x}}{s}, \frac{k_{y}}{s}\right)}{\rho^{+} s} \hat{p}_{\text {inc }^{\prime}}^{+} \\
\hat{u}_{\text {ref }, x}^{+}=\mathrm{i} \frac{k_{x}}{\rho^{+} s^{2}} \hat{p}_{\text {ref }}^{+} \quad \hat{u}_{\text {ref }, z}^{+}=\frac{\kappa^{+}\left(\frac{k_{x}}{s}, \frac{k_{y}}{s}\right)}{\rho^{+} s} \hat{p}_{\text {ref }}^{+} \\
\hat{u}_{s x}^{-}=-\mathrm{i} k_{x} \mathcal{P}_{11} \hat{\Phi}_{P f}-\mathrm{i} k_{x} \mathcal{P}_{12} \hat{\Phi}_{P s}^{-}-i s k_{x} \kappa_{S}^{-}\left(\frac{k_{x}}{s}\right) \Phi_{S}^{-} \\
\hat{u}_{s z}^{-}=s \kappa_{P f}^{-}\left(\frac{k_{x}}{s}, \frac{k_{y}}{s}\right) \mathcal{P}_{11} \hat{\Phi}_{P f}^{-}+s \kappa_{P s}^{-}\left(\frac{k_{x}}{s}, \frac{k_{y}}{s}\right) \mathcal{P}_{12} \hat{\Phi}_{P s}^{-}+\left(k_{x}^{2}+k_{y}^{2}\right) \Phi_{S}^{-}
\end{array}\right.
$$

In the following we only detail the computation of $\hat{u}_{s x, P s}^{-}=-\mathrm{i} k_{x} \mathcal{P}_{12} \hat{\Phi}_{P_{S}}^{-}$, since the computation of the other terms is very similar.

\subsection{The Laplace transform of the solution}

We apply an inverse Fourier transform in the $x$ and $y$ variable to $\hat{u}_{s x, P_{s}}^{-}$and we set $k_{x}=q_{x} s$ and $k_{y}=q_{y} s$ to obtain (we recall that we consider $y=0$ )

$$
\begin{aligned}
\tilde{u}_{s x, P s}^{-}(x, 0, z, s) & =-\int_{-\infty}^{+\infty} \int_{-\infty}^{+\infty} \frac{\mathrm{i} q_{x} \mathcal{P}_{12}}{4 \pi^{2}} \mathcal{T}_{P_{s}}\left(q_{x}, q_{y}\right) e^{\left.-s\left(-z \kappa_{P_{s}}^{-}\left(q_{x}, q_{y}\right)+h \kappa^{+}\left(q_{x}, q_{y}\right)\right)+\mathrm{i} q_{x} x\right)} d q_{x} d q_{y} \\
& =-\frac{\mathcal{P}_{12}}{4 \pi^{2}} \int_{-\infty}^{+\infty} \int_{-\infty}^{+\infty} \Xi\left(q_{x}, q_{y}\right) d q_{x} d q_{y}
\end{aligned}
$$

with

$$
\Xi\left(q_{x}, q_{y}\right)=\mathrm{i} q_{x} \mathcal{T}_{P_{s}}\left(q_{x}, q_{y}\right) e^{\left.-s\left(-z \kappa_{\mathcal{F}_{s}}^{-}\left(q_{x}, q_{y}\right)+h \kappa^{+}\left(q_{x}, q_{y}\right)\right)+\mathrm{i} q_{x} x\right)} .
$$

Let us now focus on the integral over $q_{x}$ for a fixed $q_{y}$

$$
\int_{-\infty}^{+\infty} \Xi\left(q_{x}, q_{y}\right) d q_{x}=\int_{-\infty}^{+\infty} \mathrm{i} q_{x} \mathcal{T}_{P s}\left(q_{x}, q_{y}\right) e^{\left.-s\left(-z \kappa_{P_{s}}^{-}\left(q_{x}, q_{y}\right)+h \kappa^{+}\left(q_{x}, q_{y}\right)\right)+\mathrm{i} q_{x} x\right)} d q_{x}
$$

This integral is very similar to the one we have obtained in 2D [7], therefore, using the same method, we have:

- if $\left|\gamma\left(q_{y}, t_{0}\right)\right| \leq \frac{1}{\mathcal{V}_{\max }\left(q_{y}\right)}$

$$
\int_{-\infty}^{+\infty} \Xi\left(q_{x}, q_{y}\right) d q_{x}=2 \int_{\tilde{t}_{0}\left(q_{y}\right)}^{+\infty} \Re e\left(\mathrm{i} \gamma\left(q_{y}, t\right) \mathcal{T}_{P_{s}}\left(q_{y}, \gamma\left(q_{y}, t\right)\right) \frac{\partial \gamma\left(q_{y}, t\right)}{\partial t}\right) e^{-s t} d t
$$


- if $\left|\gamma\left(q_{y}, \tilde{t}_{0}\left(q_{y}\right)\right)\right|>\frac{1}{\mathcal{V}_{\max }\left(q_{y}\right)}$

$$
\begin{aligned}
\int_{-\infty}^{+\infty} \Xi\left(q_{x}, q_{y}\right) d q_{x} & =2 \int_{\tilde{t}_{h}\left(q_{y}\right)}^{\tilde{t}_{0}\left(q_{y}\right)} \Re e\left(\mathrm{i} v\left(q_{y}, t\right) \mathcal{T}_{P s}\left(q_{y}, v\left(q_{y}, t\right)\right) \frac{\partial v\left(q_{y}, t\right)}{\partial t}\right) e^{-s t} d t \\
& +2 \int_{\tilde{t}_{0}\left(q_{y}\right)}^{+\infty} \Re e\left(\mathrm{i} \gamma\left(q_{y}, t\right) \mathcal{T}_{P s}\left(q_{y}, \gamma\left(q_{y}, t\right)\right) \frac{\partial \gamma\left(q_{y}, t\right)}{\partial t}\right) e^{-s t} d t
\end{aligned}
$$

where $\mathcal{V}_{\max }$ is greatest fictitious velocity defined by:

$$
\mathcal{V}_{\max }:=\mathcal{V}_{\max }(q)=V_{\max } \sqrt{\frac{1}{1+V_{\max }^{2} q^{2}}},
$$

$\tilde{t}_{0}$ is the fictitious arrival time of the Ps body wave we have defined in the theorem, $\tilde{t}_{h}\left(q_{y}\right)$ the fictitious arrival time of the Ps head wave defined by

$$
\tilde{t}_{h}:=\tilde{t}_{h}(q)=h \sqrt{\frac{1}{\mathcal{V}^{+2}(q)}-\frac{1}{\mathcal{V}_{\max }^{2}(q)}}-z \sqrt{\frac{1}{\mathcal{V}_{P s}^{-2}(q)}-\frac{1}{\mathcal{V}_{\max }^{2}(q)}}+\frac{|x|}{\mathcal{V}_{\max }(q)}
$$

Let us recall [16] that the condition $\left|\gamma\left(q_{y}, \tilde{t}_{0}\left(q_{y}\right)\right)\right|>\frac{1}{\mathcal{V}_{\max }\left(q_{y}\right)}$ is equivalent to

$$
\left|\gamma\left(0, t_{0}\right)\right|>\frac{1}{V_{\max }} \text { and }\left|q_{y}\right| \leq q_{\max }
$$

with

$$
q_{\max }=\sqrt{\frac{r^{2}}{\left(\frac{h}{\sqrt{\frac{1}{V^{2}}-\frac{1}{V_{\max }^{2}}}}-\frac{z}{\sqrt{\frac{1}{V_{P_{S}}^{2}}-\frac{1}{V_{\max }^{2}}}}\right)}-\frac{1}{V_{\max }^{2}} .}
$$

Moreover, $\tilde{t}_{h}$ is bijective from $\left[0 ; q_{\max }\right]$ to $\left[t_{0} ; \tilde{t}_{h}\left(q_{\max }\right)\right]$ and we denote its inverse by $q_{h}$ :

$$
q_{h}(t)=\sqrt{\frac{1}{x^{2}}\left(t+z \sqrt{\frac{1}{V_{P s}^{-2}}-\frac{1}{V_{\max }^{2}}}-h \sqrt{\frac{1}{V^{+2}}-\frac{1}{V_{\max }^{2}}}\right)^{2}-\frac{1}{V_{\max }^{2}} .}
$$

Let us also recall that for $q_{y}=q_{\max }$, the arrival times of the fictitious head and body waves are the same: $\tilde{t}_{h}\left(q_{\max }\right)=\tilde{t}_{0}\left(q_{\max }\right)$. As an illustration, we represent the functions $\tilde{t}_{0}$ (the dotted line) and $\tilde{t}_{h}$ (the solid line) in Fig. 3 .

We then deduce that 


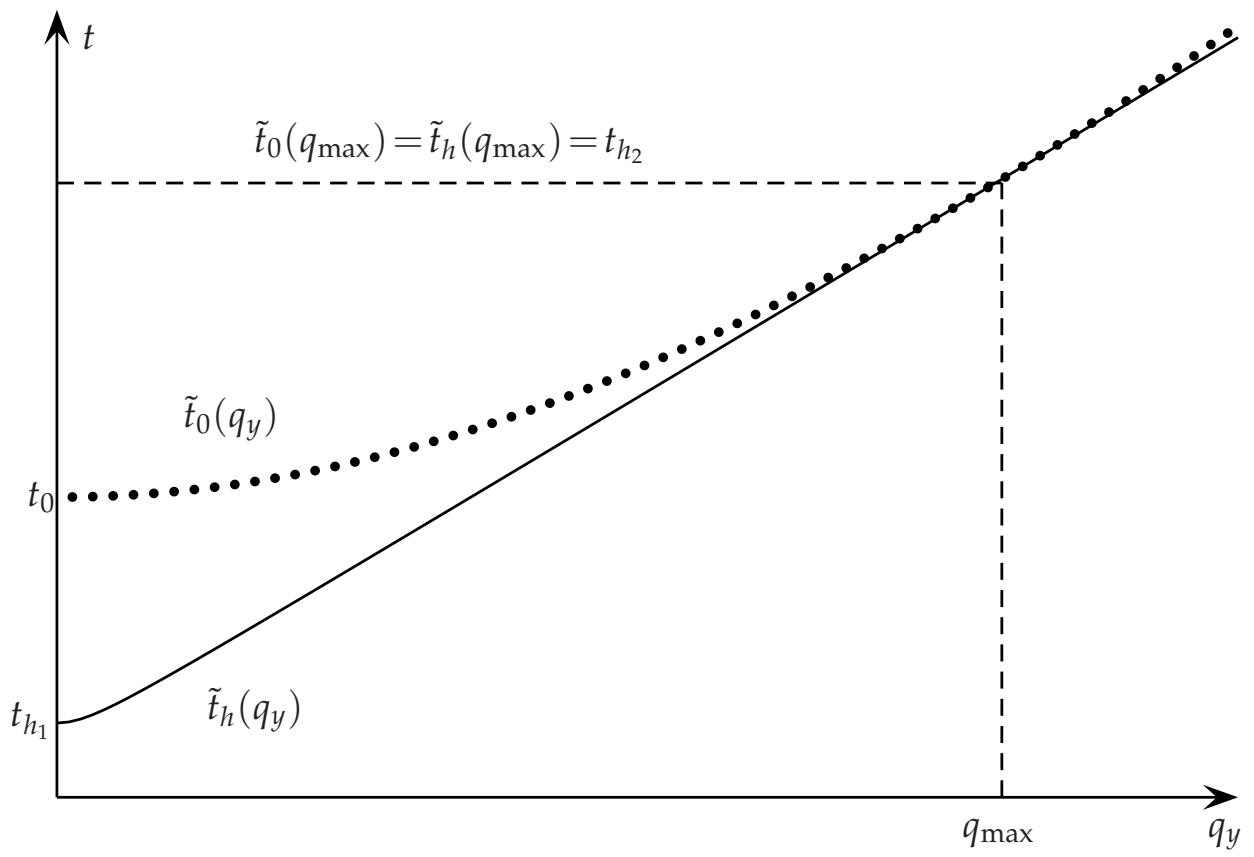

Figure 3: Functions $\tilde{t}_{0}$ (dotted line) and $\tilde{t}_{h}$ (solid line)

- if $\left|\gamma\left(0, t_{0}\right)\right| \leq \frac{1}{V_{\max }}$

$$
\int_{\mathbb{R}^{2}} \Xi\left(q_{x}, q_{y}\right) d q_{x} d q_{y}=2 \int_{\mathbb{R}} \int_{\tilde{t}_{0}\left(q_{y}\right)}^{+\infty} \Re e\left(\mathrm{i} \gamma\left(q_{y}, t\right) \mathcal{T}_{P_{s}}\left(q_{y}, \gamma\left(q_{y}, t\right)\right) \frac{\partial \gamma\left(q_{y}, t\right)}{\partial t}\right) e^{-s t} d t d q_{y}
$$

- if $\left|\gamma\left(0, t_{0}\right)\right|>\frac{1}{V_{\max }}$

$$
\begin{aligned}
\int_{\mathbb{R}^{2}} \Xi\left(q_{x}, q_{y}\right) d q_{x} d q_{y} & =2 \int_{-q_{\max }}^{+q_{\max }} \int_{\tilde{t}_{h}\left(q_{y}\right)}^{\tilde{t}_{0}\left(q_{y}\right)} \Re e\left(\mathrm{i} v\left(q_{y}, t\right) \mathcal{T}_{P_{s}}\left(q_{y}, v\left(q_{y}, t\right)\right) \frac{\partial v\left(q_{y}, t\right)}{\partial t}\right) e^{-s t} d t d q_{y} \\
& +2 \int_{\mathbb{R}} \int_{\tilde{t}_{0}\left(q_{y}\right)}^{+\infty} \Re e\left(\mathrm{i} \gamma\left(q_{y}, t\right) \mathcal{T}_{P_{s}}\left(q_{y}, \gamma\left(q_{y}, t\right)\right) \frac{\partial \gamma\left(q_{y}, t\right)}{\partial t}\right) e^{-s t} d t d q_{y}
\end{aligned}
$$

\subsection{Inversion of the integrals}

The key point of the method is the inversion of the integral with respect to $q_{y}$ with the integral with respect to $t$. For the body wave we have (see Figs. 4 and 5), after having 
remark that the integrand is even with respect to $q_{y}$ :

$$
\begin{aligned}
& \int_{-\infty}^{+\infty} \int_{\tilde{t}_{0}\left(q_{y}\right)}^{+\infty} \Re e\left(\mathrm{i} \gamma\left(q_{y}, t\right) \mathcal{T}_{P s}\left(q_{y}, \gamma\left(q_{y}, t\right)\right) \frac{\partial \gamma\left(q_{y}, t\right)}{\partial t}\right) e^{-s t} d t d q_{y} \\
= & 2 \int_{t_{0}}^{+\infty} \int_{0}^{q_{0}(t)} \Re e\left(\mathrm{i} \gamma\left(q_{y}, t\right) \mathcal{T}_{P_{s}}\left(q_{y}, \gamma\left(q_{y}, t\right)\right) \frac{\partial \gamma\left(q_{y}, t\right)}{\partial t}\right) e^{-s t} d q_{y} d t ;
\end{aligned}
$$

and for the head wave (see Figs. 6 and 7 ):

$$
\begin{aligned}
& \int_{-q_{\max }}^{+q_{\max }} \int_{\tilde{t}_{h}\left(q_{y}\right)}^{\tilde{t}_{0}\left(q_{y}\right)} \Re e\left(\mathrm{i} v\left(q_{y}, t\right) \mathcal{T}_{P_{s}}\left(q_{y}, v\left(q_{y}, t\right)\right) \frac{\partial v\left(q_{y}, t\right)}{\partial t}\right) e^{-s t} d t d q_{y} \\
= & 2 \int_{t_{h_{1}}}^{t_{0}} \int_{0}^{q_{h}(t)} \Re e\left(\mathrm{i} v\left(q_{y}, t\right) \mathcal{T}_{P_{s}}\left(q_{y}, v\left(q_{y}, t\right)\right) \frac{\partial v\left(q_{y}, t\right)}{\partial t}\right) e^{-s t} d q_{y} d t \\
+ & 2 \int_{t_{0}}^{t_{h_{2}}} \int_{q_{0}(t)}^{q_{h}(t)} \Re e\left(\mathrm{i} v\left(q_{y}, t\right) \mathcal{T}_{P_{s}}\left(q_{y}, v\left(q_{y}, t\right)\right) \frac{\partial v\left(q_{y}, t\right)}{\partial t}\right) e^{-s t} d q_{y} d t .
\end{aligned}
$$

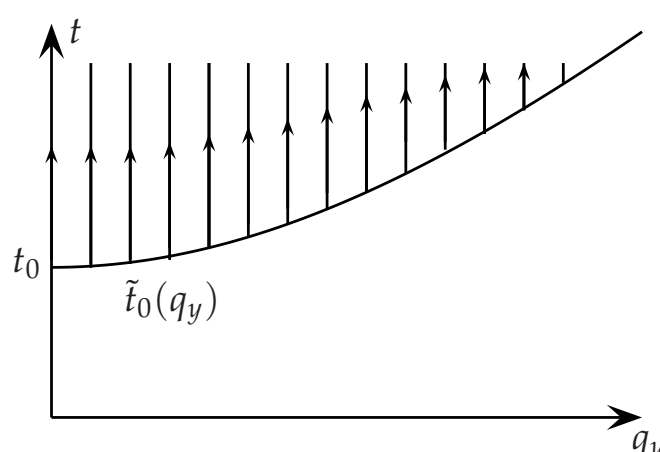

Figure 4: Integration first over $q_{y}$ then over $t$ for the body wave

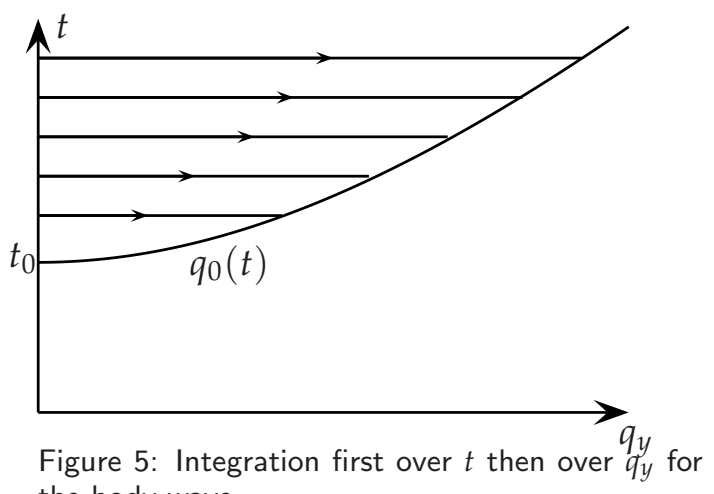
the body wave

We thus have:

$$
\tilde{u}_{s x, P s}^{-}(x, 0, z, s)=\int_{0}^{+\infty} u_{s x, P s}^{-}(x, 0, z, t) e^{-s t} d t
$$

and we conclude by using the injectivity of the Laplace transform.

\section{Numerical illustration}

To illustrate our results, we have computed the green function and the analytical solution to the following problem: we consider an acoustic layer with a density $\rho^{+}=1020 \mathrm{~kg} / \mathrm{m}^{3}$ and a celerity $V^{+}=1500 \mathrm{~m} / \mathrm{s}$ on top of a poroelastic layer whose characteristic coefficients are: 


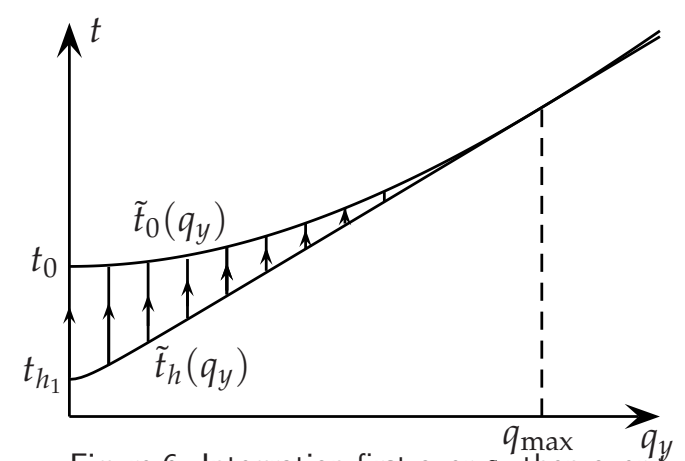

Figure 6: Integration first over $q_{y}$ thax over $q_{y}$ for the head wave

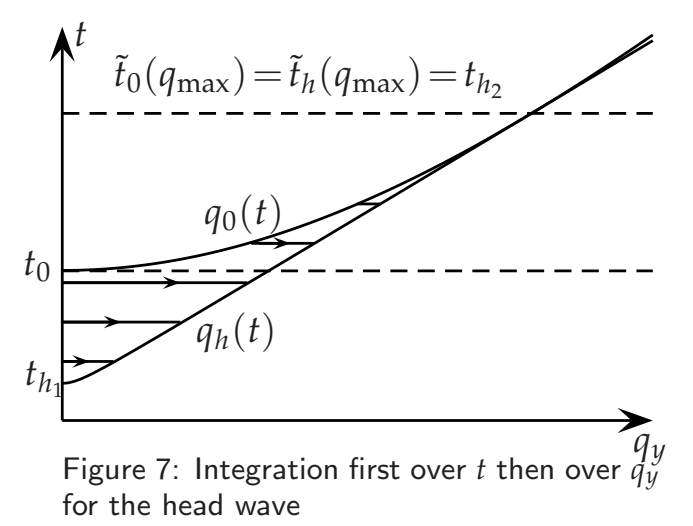
for the head wave

- the solid density $\rho_{s}^{-}=2500 \mathrm{~kg} / \mathrm{m}^{3}$;

- the fluid density $\rho_{f}^{-}=1020 \mathrm{~kg} / \mathrm{m}^{3}$;

- the porosity $\phi^{-}=0.4$;

- the tortuosity $a^{-}=2$;

- the solid bulk modulus $K_{s}^{-}=16.0554 \mathrm{GPa}$;

- the fluid bulk modulus $K_{f}^{-}=2.295 \mathrm{GPa}$;

- the frame bulk modulus $K_{b}^{-}=10 \mathrm{GPa}$;

- the frame shear modulus $\mu^{-}=9.63342 \mathrm{GPa}$;

so that the celerity of the waves in the poroelastic medium are:

- for the fast $\mathrm{P}$ wave, $V_{P f}^{-}=3677 \mathrm{~m} / \mathrm{s}$

- for the slow P wave, $V_{P s}^{-}=1060 \mathrm{~m} / \mathrm{s}$

- for the $\psi$ wave, $V_{S}^{-}=2378 \mathrm{~m} / \mathrm{s}$.

The source is located in the acoustic layer, at $500 \mathrm{~m}$ from the interface. It is a point source in space and a fifth derivative of a Gaussian of dominant frequency $f_{0}=15 \mathrm{~Hz}$ :

$$
f(t)=2 \frac{\pi^{2}}{f_{0}^{2}}\left[3+12 \frac{\pi^{2}}{f_{0}^{2}}\left(t-\frac{1}{f_{0}}\right)^{2}+4 \frac{\pi^{4}}{f_{0}^{4}}\left(t-\frac{1}{f_{0}}\right)^{4}\right] e^{-\frac{\pi^{2}}{f_{0}^{2}}\left(t-\frac{1}{f_{0}}\right)^{2}} .
$$

We compute the solution at two receivers, the first one is in the acoustic layer, at $533 \mathrm{~m}$ from the interface; the first one is in the poroelastic layer, at $533 \mathrm{~m}$ from the interface; both 
are located on a vertical line at $400 \mathrm{~m}$ from the source (see Fig. 8). To compute the integrals over $q$ and the convolution with the source function, we used a classical mid-point quadrature formula.

We represent the $z$ component of the green function associated to the displacement from $t=0$ to $t=1.2 \mathrm{~s}$ on Fig 9 and the displacement in Fig. 10. The left picture represents the solution at receiver 1 while the right picture represents the solution at receiver 2 . As all the types of waves are computed independently, it is easy to distinguish all of them, as it is indicated in the figures. solution.

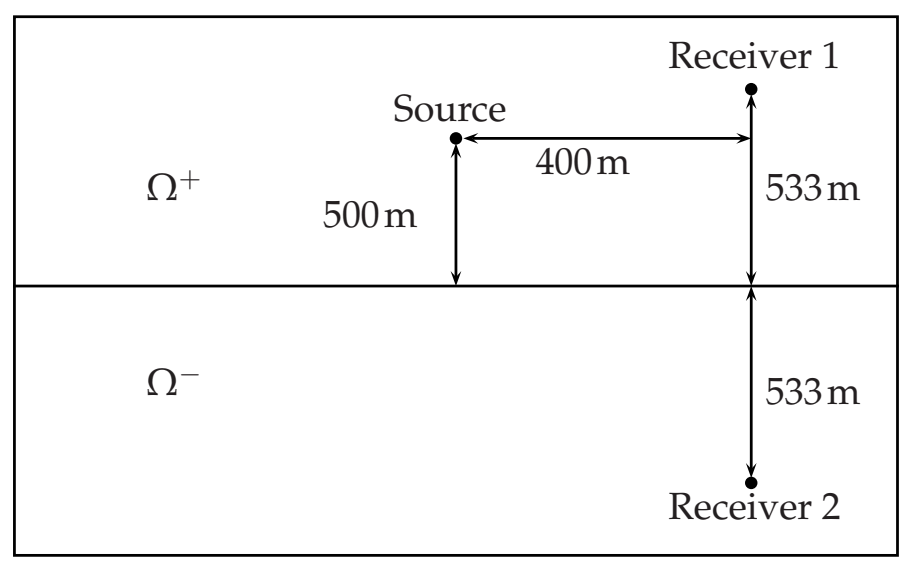

Figure 8: Configuration of the experiment
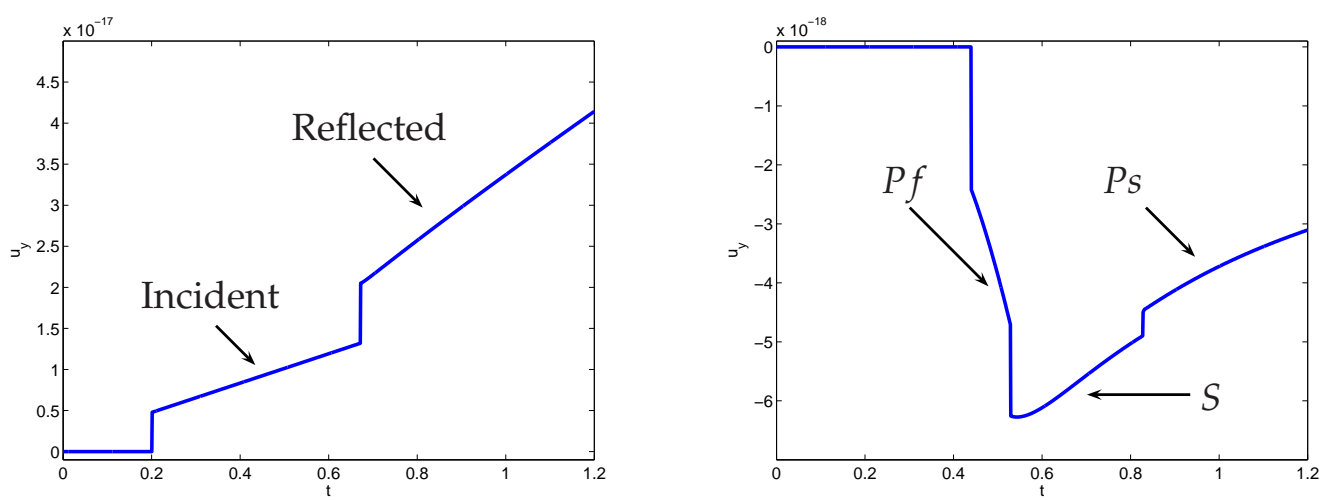

Figure 9: The $z$ component of the green function associated to the displacement at receiver 1 (left picture) and 2 (right picture). 

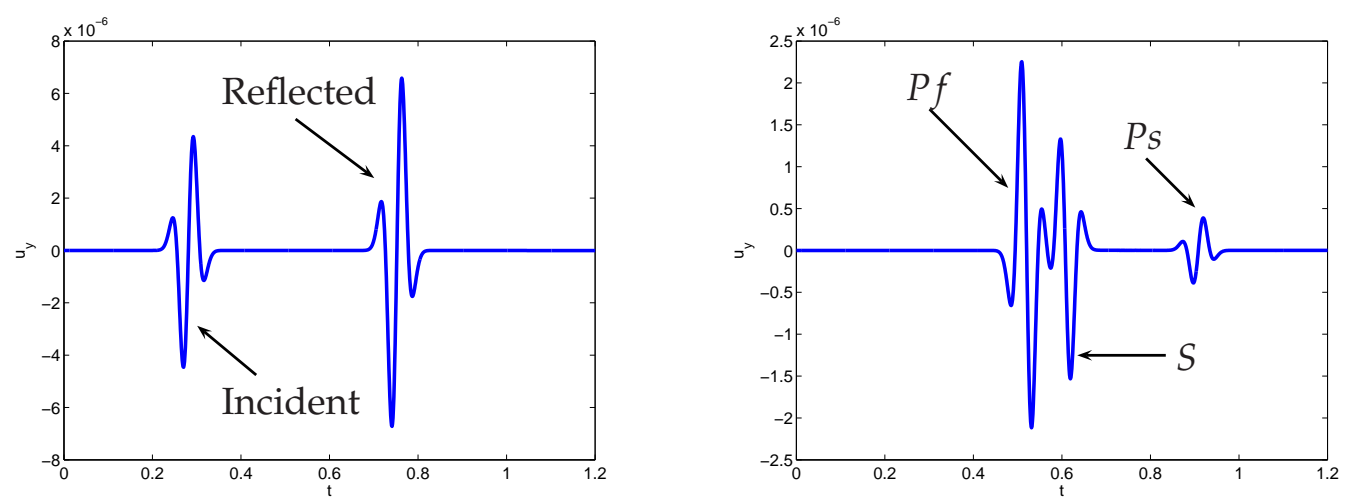

Figure 10: The $z$ component of the displacement at receiver 1 (left picture) and 2 (right picture).

\section{Conclusion}

In this paper we have provided the complete solution (reflected and transmitted wave) of the propagation of wave in a stratified 3D medium composed of an acoustic and a poroelastic layer. In a forthcoming paper we will extend the method to the propagation of waves in bilayered poroelastic medium in three dimensions.

\section{Acknowledgments}

This work was supported by the ANR project AHPI.

\section{A Definition of the fictitious and real arrival times of the body waves.}

We detail in this section the computation of the fictitious and real arrival times of the transmitted $P_{S}$ wave at point $(x, 0, z)$. For a given $q_{y} \in \mathbb{R}$, we first determine fastest path of the wave from the source to the point $(x, 0, z)$, travelling at a velocity $\mathcal{V}^{+}\left(q_{y}\right)$ in the upper layer and at a velocity $\mathcal{V}_{P_{s}}^{-}\left(q_{y}\right)$ in the bottom layer: we search a point $\xi_{0}$ on the interface between the two media which minimizes the function

$$
t(\xi)=\frac{\sqrt{\xi^{2}+h^{2}}}{\mathcal{V}^{+}\left(q_{y}\right)}+\frac{\sqrt{(x-\xi)^{2}+z^{2}}}{\mathcal{V}_{P_{s}}^{-}\left(q_{y}\right)}
$$

(see Fig. 11). This leads us to find $\xi_{0}$ such that

$$
t^{\prime}\left(\xi_{0}\right)=\frac{\xi_{0}}{\mathcal{V}^{+}\left(q_{y}\right) \sqrt{\xi_{0}^{2}+h^{2}}}+\frac{\xi_{0}-x}{\mathcal{V}_{P s}^{-}\left(q_{y}\right) \sqrt{\left(x-\xi_{0}\right)^{2}+z^{2}}}=0
$$


From a numerical point of view, the solution of this equation is done by computing the roots of the following fourth degree polynomial

$$
\begin{aligned}
& \left(\frac{1}{\mathcal{V}^{+2}\left(q_{y}\right)}-\frac{1}{\mathcal{V}_{P_{s}}^{-2}\left(q_{y}\right)}\right) X^{4}+2 x\left(\frac{1}{\mathcal{V}_{P s}^{-2}\left(q_{y}\right)}-\frac{1}{\mathcal{V}^{+2}\left(q_{y}\right)}\right) X^{3} \\
+ & \left(\frac{x^{2}+z^{2}}{\mathcal{V}^{+2}\left(q_{y}\right)}-\frac{x^{2}+h^{2}}{\mathcal{V}_{P_{s}}^{-2}\left(q_{y}\right)}\right) X^{2}+\frac{x h^{2}}{\mathcal{V}_{P_{s}}^{-2}\left(q_{y}\right)} X+\frac{x^{2} h^{2}}{\mathcal{V}_{P s}^{-2}\left(q_{y}\right)},
\end{aligned}
$$

$\xi_{0}$ is thus the only real root of this polynomial located between 0 and $x$ which is also solution of (A.1). Once $\xi_{0}$ is computed, we can define

$$
\tilde{t}_{0}\left(q_{y}\right)=\frac{\sqrt{\tilde{\zeta}_{0}^{2}+h^{2}}}{\mathcal{V}^{+}\left(q_{y}\right)}+\frac{\sqrt{\left(x-\xi_{0}\right)^{2}+z^{2}}}{\mathcal{V}_{P_{S}}^{-}\left(q_{y}\right)} \text { and } t_{0}=\tilde{t}_{0}(0)
$$

Let us remark that

Property A.1. Since the fictitious velocities are smaller than the real one, the fictitious arrival times are greater than the real one. Moreover, since the fictitious velocities are even functions decreasing on $\mathbb{R}^{+}, \tilde{t}_{0}$ is an even function, increasing on $\mathbb{R}^{+}$.

Corollaire A.1. The function $\tilde{t}_{0}$ is bijective from $\mathbb{R}^{+}$to $\mathbb{R}^{+}$.

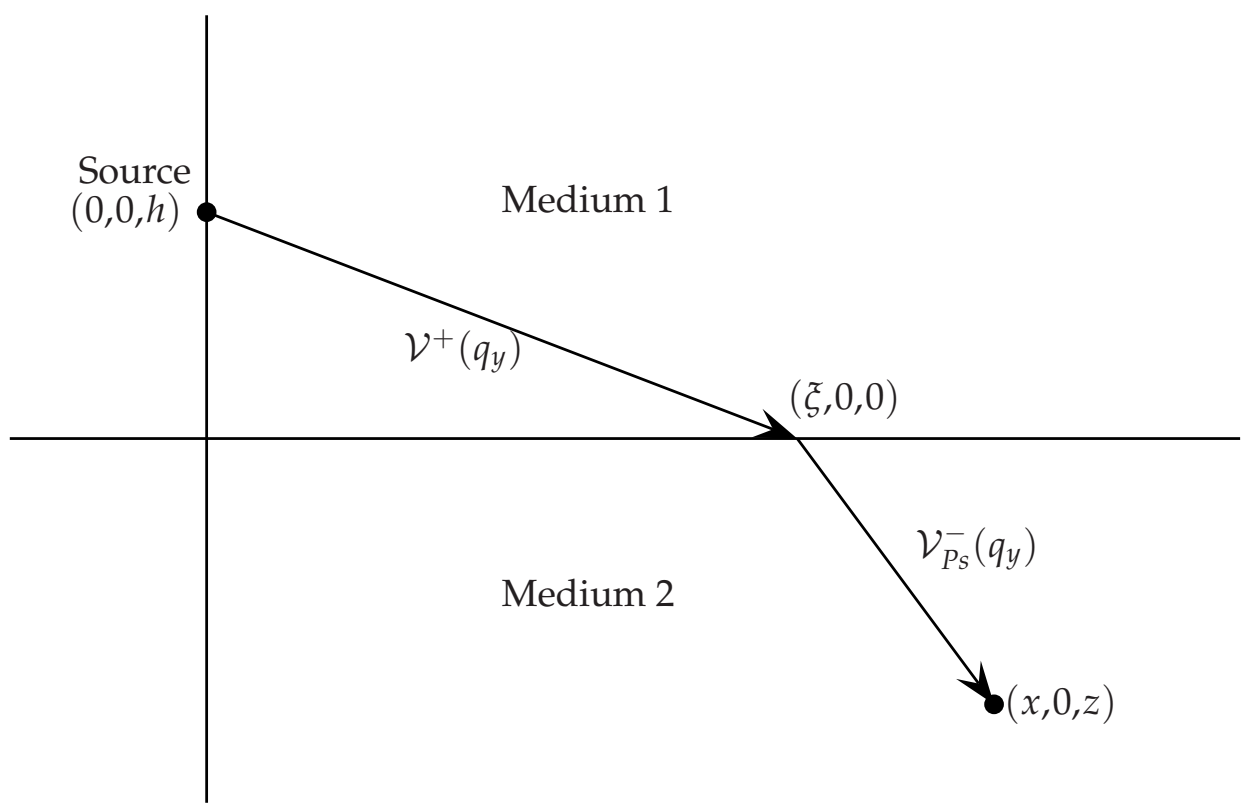

Figure 11: Path of the transmitted $i$ wave 


\section{References}

[1] L. Cagniard, Reflection and refraction of progressive seismic waves, McGraw-Hill, 1962.

[2] A. T. de Hoop, The surface line source problem, Appl. Sci. Res. B., 8(1959), 349-356.

[3] A. Ezziani, Modélisation mathématique et numérique de la propagation d'ondes dans les milieux viscoélastiques et poroélastiques, Ph.D. Thesis, Université Paris 9, 2005, in french.

[4] S. Feng and D. L. Johnson, High-frequency acoustic properties of a fluid/porous solid interface. II. The 2D reflection Green's function, J. Acoust. Sec. Am., 74(1983), 915-924.

[5] J. Diaz and A. Ezziani, Gar6more 2D, http://www.spice-rtn.org/library/software/Gar6more2D, 2008.

[6] J. Diaz and A. Ezziani, Gar6more 3D, http://www.spice-rtn.org/library/software/Gar6more3D, 2008.

[7] J. Diaz and A. Ezziani, Analytical Solution for Wave Propagation in Stratified Acoustic/Porous Media. Part I: the 2D Case, Technical Report 6509, INRIA, 2008.

[8] J. Diaz and A. Ezziani, Analytical Solution for Wave Propagation in Stratified Poroelastic Medium. Part I: the 2D Case, Technical Report 6591, INRIA, 2008.

[9] M. A. Biot, Theory of propagation of elastic waves in a fluid-saturated porous solid. I. Lowfrequency range, J. Acoust. Soc. Am, 28(1956), 168-178.

[10] M. A. Biot, Theory of propagation of elastic waves in a fluid-saturated porous solid. II. Higher frequency range, J. Acoust. Soc. Am, 28(1956), 179-191.

[11] M. A. Biot, Mechanics of deformation and acoustic propagation in porous media, J. Appl. Phys., 33(1962), 1482-1498.

[12] J. M. Carcione, Wave Fields in Real Media: Wave propagation in Anisotropic, Anelastic and Porous Media, Pergamon, 2001.

[13] J. H. M. T. van der Hijden, Propagation of transient elastic waves in stratified anisotropic media, 32 volume of North Holland Series in Applied Mathematics and Mechanics, Elsevier Science Publishers, 1987.

[14] Y. Pao and R. Gajewski, The generalized ray theory and transient response of layered elastic solids, volume 13 of Physical Acoustics, chapter 6, 183-265, 1977.

[15] Q. Grimal, Etude dans le domaine temporel de la propagation d'ondes elastiques en milieux stratifis ; modélisation de la reponse du thorax a un impacts, Ph.D. Thesis, Université Paris12-Val de Marne, 2003, in french.

[16] J. Diaz, Approches analytiques et numériques de problèmes de transmission en propagation d'ondes en régime transitoire. Application au couplage fluide-structure et aux méthodes de couches parfaitement adaptées, Ph.D Thesis, Université Paris 6, 2005, in french. 